\title{
New Hermite-Hadamard and Jensen inequalities for log-s-convex fuzzy-interval-valued functions in the second sense
}

\author{
Peide Liu ${ }^{1}$ Muhammad Bilal Khan ${ }^{2}$ (D) Muhammad Aslam Noor ${ }^{2} \cdot$ Khalida Inayat Noor $^{2}$
}

Received: 12 January 2021 / Accepted: 12 April 2021 / Published online: 13 May 2021

(c) The Author(s) 2021

\begin{abstract}
In this paper, our aim is to consider the new class of log-convex fuzzy-interval-valued function known as log-s-convex fuzzyinterval-valued functions (log-s-convex fuzzy-IVFs). By this concept, we have introduced Hermite-Hadamard inequalities (HH-inequalities) by means of fuzzy order relation. This fuzzy order relation is defined level-wise through Kulisch-Miranker order relation defined on interval space. Moreover, some new Hermite-Hadamard-Fejér inequalities (HH-Fejér-inequalities) and Jensen's inequalities via log-s-convex fuzzy-IVFs are also established and verified with the support of useful examples. Some special cases are also discussed which can be viewed as applications of fuzzy-interval HH-inequalities. The concepts and approaches of this paper may be the starting point for further research in this area.
\end{abstract}

Keywords Fuzzy-interval-valued functions · Log-s-convex fuzzy-interval-valued function · Hermite-Hadamard inequality · Hermite-Hadamard-Fejér inequality $\cdot$ Jensen's inequality

Mathematics Subject Classification $26 \mathrm{~A} 33 \cdot 26 \mathrm{~A} 51 \cdot 26 \mathrm{D} 10$

\section{Introduction}

In last few decades much attention has been given in studying and distinguishing diverse directions of classical idea of convexity. Recently, many extensions and generalizations of convex functions have been established. For more useful details, see [1-3] and the references are therein. In classical approach, a real valued function $\mathcal{F}: K \rightarrow \mathbb{R}$ is called convex if

Muhammad Bilal Khan bilal42742@gmail.com

Peide Liu

peide.liu@gmail.com

Muhammad Aslam Noor

aslamnoor@comsats.edu.pk

Khalida Inayat Noor

khalidanoor@hotmail.com

1 School of Management Science and Engineering, Shandong University of Finance and Economics, Jinan 250014, Shandong, China

2 Department of Mathematics, COMSATS University Islamabad, Islamabad, Pakistan
$\mathcal{F}(\tau x+(1-\tau) y) \leq \tau \mathcal{F}(x)+(1-\tau) \mathcal{F}(y)$,

for all $x, y \in K, \tau \in[0,1]$.

The concept of convexity with integral problem is an interesting area for research. Therefore, many inequalities have been introduced as applications of convex functions. Among those, the Hermite-Hadamard inequality (HH-inequality) is an interesting outcome in convex analysis. The HH-inequality $[4,5]$ for convex function $\mathcal{F}: K \rightarrow \mathbb{R}^{+}$on an interval $K=[u, \vartheta]$

$\mathcal{F}\left(\frac{u+\vartheta}{2}\right) \leq \frac{1}{\vartheta-u} \int_{u}^{\vartheta} \mathcal{F}(x) \mathrm{d} x \leq \frac{\mathcal{F}(u)+\mathcal{F}(\vartheta)}{2}$,

for all $u, \vartheta \in K$.

It is well known that log-convex functions have serious importance in convex theory because using these functions we can derive more accurate inequalities as compared to convex functions. Recently, some of the authors discussed different classes of log-convex and log-nonconvex functions, for example $h$-convexity [6], $s$-logarithmically convexity [7], and log-preinvexity [8]. Specifically, an important special class of convex functions is the log-convex functions proposed by Pečarić et al. [9]. A step forward, Noor et al. 
[10] also presented the class of log- $h$-convex functions and constructed the following $\mathrm{HH}$-inequality for $\log -h$-convex functions:

Let $\mathcal{F}: K \rightarrow \mathbb{R}^{+}$be a log- $h$-convex function on a $h$ convex set $K$ with $h:[0,1] \subseteq K \rightarrow \mathbb{R}^{+}$such that $\not \equiv 0$ and $u, \vartheta \in K$ with $u \leq \vartheta$. Then,

$\mathcal{F}\left(\frac{u+\vartheta}{2}\right)^{\frac{1}{2 h\left(\frac{1}{2}\right)}} \leq \exp \left[\frac{1}{\vartheta-u} \int_{u}^{\vartheta} \ln \mathcal{F}(x) \mathrm{d} x\right] \leq[\mathcal{F}(u) \mathcal{F}(\vartheta)]^{\int}{ }_{0}^{1} h(\tau) \mathrm{d} \tau$

If $\mathcal{T}$ is concave then, inequality (3) is reversed. If $h(\tau)=\tau^{s}$ with $s \in(0,1)$ then, inequality (3) reduces for $\log$-s-With the assistance of inequality (3), some classical inequalities can be obtained through special log-convex function. In addition, these inequalities have a very significant role for log-convex functions in both pure and applied mathematics.

Jensen's inequality [11] is one of these inequalities for convex functions, which can be stated as follows.

Let $\omega_{j} \in[0,1], u_{j} \in[u, \vartheta],(j=1,2,3, \ldots k, k \geq 2)$ and $\mathcal{T}$ be a convex function then,

$\mathcal{T}\left(\sum_{j=1}^{k} \omega_{j} x_{j}\right) \preccurlyeq\left(\sum_{j=1}^{k} \omega_{j} \mathcal{T}\left(x_{j}\right)\right)$,

with $\sum_{j=1}^{k} \omega_{j}=1$. If $\mathcal{T}$ is concave then, inequality (4) is reversed.

Moreover, with the help of this concept, some Jensen's and HH-inequalities are defined [12-16].

On the other hand, the concept of interval analysis was proposed and investigated by Moore [17] and Kulish and Miranker [18]. It is a discipline in which an uncertain variable is represented by an interval of real numbers. Inspired by the above literature, in 2018, Guo et al. [19] introduced log- $h$ -convex interval-valued functions (in short, log- $h$-convexIVF) and prove the HH-inequality for log- $h$-convex IVFs as follows:

Let $\mathcal{F}:[u, \vartheta] \subset \mathbb{R} \rightarrow \mathcal{K}_{C}{ }^{+}$be a log- $h$-convex-IVF given by $\mathcal{F}(x)=\left[\mathcal{F}_{*}(x), \mathcal{F}^{*}(x)\right]$ for all $x \in[u, \vartheta]$, where $\mathcal{F}_{*}(x)$ is a $\log$ - $h$-convex function and $\mathcal{F}^{*}(x)$ is a $\log$ - $h$-concave function. If $\mathcal{F}$ is Riemann integrable, then

$\mathcal{F}\left(\frac{u+\vartheta}{2}\right)^{\frac{1}{2 h\left(\frac{1}{2}\right)}} \supseteq \exp \left[\frac{1}{\vartheta-u} \int_{u}^{\vartheta} \ln \mathcal{F}(x) \mathrm{d} x\right] \supseteq[\mathcal{F}(u) \mathcal{F}(\vartheta)]^{\int_{0}^{1} h(\tau) \mathrm{d} \tau}$

We urge readers to make further analysis on literature about applications and properties of generalized convex functions and $\mathrm{HH}$-integral inequalities [20-25] and the references therein.

In [26], the enormous research work fuzzy set and system has been dedicated on development of different fields and it plays an important role in the study of a wide class problems arising in pure mathematics and applied sciences including operation research, computer science, managements sciences, artificial intelligence, control engineering and decision sciences. Similarly, the notions of convexity and non-convexity play a vital role in optimization under fuzzy domain because during characterization of the optimality condition of convexity, we obtain fuzzy variational inequalities so variational inequality theory and fuzzy complementary problem theory established powerful mechanism of the mathematical problems and they have a friendly relationship. Many authors contributed to this fascinating and interesting field. Besides, Nanda and Kar [27], and Chang [28] discussed the idea of convex fuzzy mapping and find its optimality condition with the support of fuzzy variational inequality. Generalization and extension of fuzzy convexity play a vital and significant implementation in diverse directions. So let's note that, one of the most considered classes of nonconvex fuzzy mapping is preinvex fuzzy mapping. Noor [29] introduced this idea and proved some results that distinguish the fuzzy optimality condition of differentiable fuzzy preinvex mappings by fuzzy variational-like inequality. We refer to the readers for further analysis of literature on the applications and properties of variational-like inequalities and generalized convex fuzzy mappings [30-35] and the references therein. The fuzzy mappings are fuzzy-interval valued functions (fuzzyIVFs, in short). There are some integrals to deal with fuzzyIVFs, where the integrands are fuzzy-IVFs. For instance, Osuna-Gómez et al. [36], and Costa [37] constructed Jensen's integral inequality for fuzzy-IVF. Using the same approach Costa and Floures presented Minkowski and Beckenbach's inequalities, where the integrands are fuzzy-IVFs. Motivated by $[19,37-39]$ and especially by Costa and Roman-Flores [40] because they established the relation between elements of fuzzy-interval space and interval space, and introducing level-wise fuzzy order relation on fuzzy-interval space through Kulisch-Miranker order relation defined on interval space. Using this concept on fuzzy-interval space, we generalize integral inequalities (3), (4) and (5) by constructing fuzzy-interval integral inequality for convex fuzzy-IVF, where the integrands are convex fuzzy-IVF.

Inspired by the ongoing research work, we have considered the class of generalized log-convex fuzzy-IVFs is known as log-s-convex fuzzy-IVFs. Using this class, we obtain fuzzy-interval $\mathrm{HH}$-inequalities and verify these inequalities with the support of examples. Moreover, some Jensen's inequalities are also introduced via log-s-convex fuzzy-IVFs.

\section{Preliminaries}

Let $\mathcal{K}_{C}$ be the collection of all closed and bounded intervals of $\mathbb{R}$ that is $\mathcal{K}_{C}=\left\{\left[\omega_{*}, \omega^{*}\right]: \omega_{*}, \omega^{*} \in \mathbb{R}\right.$ and $\left.\omega_{*} \leq \omega^{*}\right\}$. If $\omega_{*} \geq 0$, then $\left[\omega_{*}, \omega^{*}\right]$ is called positive interval. The set 
of all positive interval is denoted by $\mathcal{K}_{C}^{+}$and defined as $\mathcal{K}_{C}^{+}=\left\{\left[\omega_{*}, \omega^{*}\right]:\left[\omega_{*}, \omega^{*}\right] \in \mathcal{K}_{C}\right.$ and $\left.\omega_{*} \geq 0\right\}$.

We now discuss some properties of intervals under the arithmetic operations addition, multiplication and scalar multiplication. If $\left[\mu_{*}, \mu^{*}\right],\left[\omega_{*}, \omega^{*}\right] \in \mathcal{K}_{C}$ and $\rho \in \mathbb{R}$, then arithmetic operations are defined by

$\left[\mu_{*}, \mu^{*}\right]+\left[\omega_{*}, \omega^{*}\right]=\left[\mu_{*}+\omega_{*}, \mu^{*}+\omega^{*}\right]$,

$\left[\mu_{*}, \mu^{*}\right] \times\left[\omega_{*}, \omega^{*}\right]=\left[\min \left\{\mu_{*} \omega_{*}, \mu^{*} \omega_{*}, \mu_{*} \omega^{*}, \mu^{*} \omega^{*}\right\}, \max \left\{\mu_{*} \omega_{*}, \mu^{*} \omega_{*}, \mu_{*} \omega^{*}, \mu^{*} \omega^{*}\right\}\right]$,

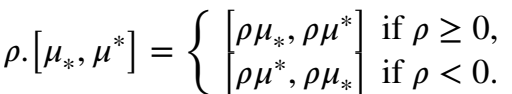

For $\left[\mu_{*}, \mu^{*}\right],\left[\omega_{*}, \omega^{*}\right] \in \mathcal{K}_{C}$, the inclusion " $\subseteq$ " is defined by

$\left[\mu_{*}, \mu^{*}\right] \subseteq\left[\omega_{*}, \omega^{*}\right]$, if and only if $\omega_{*} \leq \mu_{*}, \mu^{*} \leq \omega^{*}$

Remark 2.1 The relation " $\leq_{I}$ " defined on $\mathcal{K}_{C}$ by

$\left[\mu_{*}, \mu^{*}\right] \leq_{I}\left[\omega_{*}, \omega^{*}\right]$ if and only if $\mu_{*} \leq \omega_{*}, \mu^{*} \leq \omega^{*}$,

for all $\left[\mu_{*}, \mu^{*}\right],\left[\omega_{*}, \omega^{*}\right] \in \mathcal{K}_{C}$, it is an order relation, see [17]. For given $\left[\mu_{*}, \mu^{*}\right],\left[\omega_{*}, \omega^{*}\right] \in \mathcal{K}_{C}$, we say that $\left[\mu_{*}, \mu^{*}\right] \leq_{I}\left[\omega_{*}, \omega^{*}\right]$ if and only if $\mu_{*} \leq \omega_{*}, \mu^{*} \leq \omega^{*}$ or $\mu_{*} \leq \omega_{*}, \mu^{*}<\omega^{*}$.

The concept of Riemann integral for IVF first introduced by Moore [16] is defined as follow:

Theorem 2.2 [16] If $\mathcal{F}:[c, d] \subset \mathbb{R} \rightarrow \mathcal{K}_{C}$ is an IVF on such that $\left[\mathcal{F}_{*}, \mathcal{F}^{*}\right]$. Then $\mathcal{F}$ is Riemann integrable over. $[c, d]$ if and only if, $\mathcal{F}_{*}$ and $\mathcal{F}^{*}$ both are Riemann integrable over: $[c, d]$ such that

(IR) $\int_{c}^{d} \mathcal{F}(x) \mathrm{d} x=\left[(R) \int_{c}^{d} \mathcal{F}_{*}(u) \mathrm{d} x,(R) \int_{c}^{d} \mathcal{F}^{*}(u) \mathrm{d} x\right]$.

The collection of all Riemann integrable real valued functions and Riemann integrable IVFs is denoted by $\mathcal{R}_{[c, d]}$ and $\mathcal{I R}_{[c, d]}$, respectively.

Let $\mathbb{R}$ be the set of real numbers. A fuzzy subset set $\mathcal{A}$ of $\mathbb{R}$ is distinguished by a function $\varphi: \mathbb{R} \rightarrow[0,1]$ called the membership function. In this study this depiction is approved. Moreover, the collection of all fuzzy subsets of $\mathbb{R}$ is denoted by $\mathbb{F}(\mathbb{R})$.

A real fuzzy-interval $\varphi$ is a fuzzy set in $\mathbb{R}$ with the following properties:

(1) $\varphi$ is normal i.e. there exists $x \in \mathbb{R}$ such that $\varphi(x)=1$;
(2) $\varphi$ is upper semi continuous i.e., for given $x \in \mathbb{R}$, for every $x \in \mathbb{R}$ there exist $\varepsilon>0$ there exist $\delta>0$ such that $\varphi(x)-\varphi(y)<\varepsilon$ for all $y \in \mathbb{R}$ with $|x-y|<\delta$.

(3) $\varphi$ is fuzz y convex i.e., $\varphi((1-\tau) x+\tau y) \geq \min (\varphi(x), \varphi(y)), \quad \forall x, y \in \mathbb{R} \quad$ and $\tau \in[0,1]$

(4) $\varphi$ is compactly supported i.e., $\operatorname{cl}\{x \in \mathbb{R}|\varphi(x)\rangle 0\}$ is compact.
The collection of all real fuzzy-intervals is denoted by $\mathbb{F}_{C}(\mathbb{R})$.

Since $\mathbb{F}_{C}(\mathbb{R})$ denotes the set of all real fuzzy-intervals and let $\varphi \in \mathbb{F}_{C}(\mathbb{R})$ be real fuzzy-interval, if and only if, $\alpha$-levels $[\varphi]^{\alpha}$ is a nonempty compact convex set of $\mathbb{R}$. This is represented by

$[\varphi]^{\alpha}=\{x \in \mathbb{R} \mid \varphi(x) \geq \alpha\}$,

from these definitions, we have

$[\varphi]^{\alpha}=\left[\varphi_{*}(\alpha), \varphi^{*}(\alpha)\right]$,

where

$\varphi_{*}(\alpha)=\inf \{x \in \mathbb{R} \mid \varphi(x) \geq \alpha\}, \quad \varphi^{*}(\alpha)=\sup \{x \in \mathbb{R} \mid \varphi(x) \geq \alpha\}$.

Thus a real fuzzy-interval $\varphi$ can be identified by parameterized triples

$\left\{\left(\varphi_{*}(\alpha), \varphi^{*}(\alpha), \alpha\right): \alpha \in[0,1]\right\}$.

This leads the following characterization of a real fuzzyinterval in terms of the two end point functions $\varphi_{*}(\alpha)$ and $\varphi^{*}(\alpha)$.

Theorem $2.3[31,38]$ Suppose that $\varphi_{*}(\alpha):[0,1] \rightarrow \mathbb{R}$ and $\varphi^{*}(\alpha):[0,1] \rightarrow \mathbb{R}$ satisfy the following conditions:

(1) $\varphi_{*}(\alpha)$ is a non-decreasing function.

(2) $\varphi^{*}(\alpha)$ is a non-increasing function.

(3) $\varphi_{*}(1) \leq \varphi^{*}(1)$.

(4) $\varphi_{*}(\alpha)$ and $\varphi^{*}(\alpha)$ are bounded and left continuous on $(0,1]$ and right continuous at $\alpha=0$.

Moreover, if $\varphi: \mathbb{R} \rightarrow[0,1]$ is a real fuzzy-interval given by $\left[\varphi_{*}(\alpha), \varphi^{*}(\alpha)\right]$, then function $\varphi_{*}(\alpha)$ and $\varphi^{*}(\alpha)$ find the conditions (1)-(4).

Proposition 2.4 [40] Let $\varphi, \phi \in \mathbb{F}_{C}(\mathbb{R})$. Then relation " $\preccurlyeq$ " given on $\mathbb{F}_{C}(\mathbb{R})$ by

$\varphi \preccurlyeq \phi$ if and only if, $[\varphi]^{\alpha} \leq_{I}[\phi]^{\alpha}$ for all $\alpha \in[0,1]$, 
it is partial order relation.

We now discuss some properties of real fuzzy-intervals under addition, scalar multiplication, multiplication and division. If $\varphi, \phi \in \mathbb{F}_{C}(\mathbb{R})$ and $\rho \in \mathbb{R}$, then arithmetic operations are defined by

$[\varphi \tilde{+} \phi]^{\alpha}=[\varphi]^{\alpha}+[\phi]^{\alpha}$,

$[\varphi \tilde{\times} \phi]^{\alpha}=[\varphi]^{\alpha} \times[\phi]^{\alpha}$,

$[\rho \cdot \varphi]^{\alpha}=\rho \cdot[\varphi]^{\alpha}$

For $\psi \in \mathbb{F}_{C}(\mathbb{R})$ such that $\varphi=\phi \tilde{+} \psi$, then by this result we have existence of Hukuhara difference of $\varphi$ and $\phi$, and we say that $\psi$ is the H-difference of $\varphi$ and $\phi$, and denoted by $\varphi \simeq \phi$. If H-difference exists, then

$(\psi)^{*}(\alpha)=(\varphi \tilde{\sim} \phi)^{*}(\alpha)=\varphi^{*}(\alpha)-\phi^{*}(\alpha)$,

$(\psi)_{*}(\alpha)=(\varphi \tilde{\sim} \phi)_{*}(\alpha)=\varphi_{*}(\alpha)-\phi_{*}(\alpha)$.

Remark 2.5 Obviously, $\mathbb{F}_{C}(\mathbb{R})$ is closed under addition and nonnegative scaler multiplication and above defined properties on $\mathbb{F}_{C}(\mathbb{R})$ are equivalent to those derived from the usual extension principle. Furthermore, for each scalar number $\rho \in \mathbb{R}$

$[\rho \tilde{+} \varphi]^{\alpha}=\rho+[\varphi]^{\alpha}$.

Definition 2.6 [3] A fuzzy-interval-valued map $\mathcal{F}: K \subset \mathbb{R} \rightarrow \mathbb{F}_{C}(\mathbb{R})$ is called fuzzy-IVF. For each $\alpha \in[0,1]$, whose $\alpha$-levels define the family of IVFs $\mathcal{F}_{\alpha}: K \subset \mathbb{R} \rightarrow \mathcal{K}_{C}$ are given by $\mathcal{F}_{\alpha}(x)=\left[\mathcal{F}_{*}(x, \alpha), \mathcal{F}^{*}(x, \alpha)\right]$ for all $x \in K$. Here, for each $\alpha \in[0,1]$, the end point real functions $\mathcal{F}_{*}(., \alpha), \mathcal{F}^{*}(., \alpha): K \rightarrow \mathbb{R}$ are called lower and upper functions of $\mathcal{F}$.

Remark 2.7 Let $\mathcal{F}: K \subset \mathbb{R} \rightarrow \mathbb{F}_{C}(\mathbb{R})$ be a fuzzy-IVF. Then $\mathcal{F}(x)$ is said to be continuous at $x \in K$, if for each $\alpha \in[0,1]$, both end point functions $\mathcal{F}_{*}(x, \alpha)$ and $\mathcal{F}^{*}(x, \alpha)$ are continuous at $x \in K$.

From above literature review, following results can be concluded, see [3, 16, 38, 40]:

Definition 2.8 Let $\mathcal{F}:[c, d] \subset \mathbb{R} \rightarrow \mathbb{F}_{C}(\mathbb{R})$ is called fuzzy-IVF. The fuzzy integral of $\mathcal{F}$ over $[c, d]$, denoted by $(F R) \int_{c}^{d} \mathcal{F}(x) \mathrm{d} x$, it is defined level-wise by

$$
\begin{gathered}
{\left[(F R) \int_{c}^{d} \mathcal{F}(x) \mathrm{d} x\right]^{\alpha}=(I R) \int_{c}^{d} \mathcal{F}_{\alpha}(x) \mathrm{d} x} \\
\quad=\left\{\int_{c}^{d} \mathcal{F}(x, \alpha) \mathrm{d} x: \mathcal{F}(x, \alpha) \in \mathcal{R}_{[c, d]}\right\},
\end{gathered}
$$

for all $\alpha \in[0,1]$, where $\mathcal{R}_{[c, d]}$ is the collection of end point functions of IVFs. $\mathcal{F}$ is $(F R)$-integrable over $[c, d]$ if $(F R) \int_{c}^{d} \mathcal{F}(x) \mathrm{d} x \in \mathbb{F}_{C}(\mathbb{R})$. Note that, if both end point functions are Lebesgue-integrable, then $\mathcal{F}$ is fuzzy Aumannintegrable, see [3].

Theorem 2.9 Let $\mathcal{F}:[c, d] \subset \mathbb{R} \rightarrow \mathbb{F}_{C}(\mathbb{R}) \quad b e$ a fuzzy-IVF, whose $\alpha$-levels define the family of IVFs $\mathcal{F}_{\alpha}:[c, d] \subset \mathbb{R} \rightarrow \mathcal{K}_{C}$ are given by $\mathcal{F}_{\alpha}(x)=\left[\mathcal{F}_{*}(x, \alpha), \mathcal{F}^{*}(x, \alpha)\right]$ for all $x \in[c, d]$ and for all $\alpha \in[0,1]$. Then $\mathcal{F}$ is $(F R)$-integrable over $[c, d]$ if and only if, $\mathcal{F}_{*}(x, \alpha)$ and $\mathcal{F}^{*}(x, \alpha)$ both are $R$-integrable over $[c, d]$. Moreover, if $\mathcal{F}$ is $(F R)$-integrable over $[c, d]$, then

$$
\begin{aligned}
& {\left[(F R) \int_{c}^{d} \mathcal{F}(x) \mathrm{d} x\right]^{\alpha}} \\
& \quad=\left[(R) \int_{c}^{d} \mathcal{F}_{*}(x, \alpha) \mathrm{d} x,(R) \int_{c}^{d} \mathcal{F}^{*}(x, \alpha) \mathrm{d} x\right] \\
& =(I R) \int_{c}^{d} \mathcal{F}_{\alpha}(x) \mathrm{d} x
\end{aligned}
$$

for all $\alpha \in[0,1]$.

The family of all (FR)-integrable fuzzy-IVFs and $R$-integrable functions over $[c, d]$ are denoted by $\mathcal{F} \mathcal{R}_{([c, d], \alpha)}$ and $\mathcal{R}_{([c, d], \alpha)}$, for all $\alpha \in[0,1]$.

Definition 2.10 [7] A function $\mathcal{T}: K \rightarrow \mathbb{R}$ is said to be $\log -s$-convex function in the second sense if

$$
\mathcal{T}(\tau x+(1-\tau) y) \leq \mathcal{T}(x)^{\tau^{s}} \mathcal{T}(y)^{(1-\tau)^{s}}, \quad \forall x, y \in K, \tau \in[0,1]
$$

where $\mathcal{T}(x) \geq 0, s \in[0,1]$. If (13) is reversed then, $\mathcal{T}$ is called log-s-concave in the second sense.

Definition 2.11 [9] A function $\mathcal{T}: K \rightarrow \mathbb{R}$ is said to be log-convex function if

$$
\mathcal{T}(\tau x+(1-\tau) y) \leq \mathcal{T}(x)^{\tau} \mathcal{T}(y)^{1-\tau}, \quad \forall x, y \in K, \tau \in[0,1]
$$

where $\mathcal{T}(x) \geq 0$. If (14) is reversed then, $\mathcal{T}$ is called log-concave. 
Definition 2.12 [10] A function $\mathcal{T}: K \rightarrow \mathbb{R}$ is said to be $\log P$-convex function if

$\mathcal{T}(\tau x+(1-\tau) y) \leq \mathcal{T}(x) \mathcal{T}(y), \quad \forall x, y \in K, \tau \in[0,1]$,

where $\mathcal{T}(x) \geq 0$. If (15) is reversed then, $\mathcal{T}$ is called log- $P$ -concave.

Now we define the new class, log-s-convex fuzzy-IVF in the second by means of fuzzy order relation.

Definition 2.13 Let $K$ be a convex set. Then fuzzy-IVF $\mathcal{T}: K \rightarrow \mathbb{F}_{C}(\mathbb{R})$ is said to be:

- $\log$-s-convex fuzzy-IVF in the second sense on $K$ if

$$
\mathcal{T}(\tau x+(1-\tau) y) \leqslant \mathcal{T}(x)^{\tau^{s}} \tilde{x} \mathcal{T}(y)^{(1-\tau)^{s}},
$$

for all $x, y \in K, \tau \in[0,1]$, where $\mathcal{T}(x) \geqslant \tilde{0}, s \in[0,1]$.

- log-s-concave fuzzy-IVF in the second sense on $K$ if inequality (16) is reversed.

- Affine log-s-convex fuzzy-IVF in the second sense on $K$ if

$$
\mathcal{T}(\tau x+(1-\tau) y)=\mathcal{T}(x)^{\tau^{s}} \tilde{x} \mathcal{T}(y)^{(1-\tau)^{s}},
$$

for all $x, y \in K, \tau \in[0,1]$, where $\mathcal{T}(x) \geqslant \tilde{0}, s \in[0,1]$.

The following remark discusses the classical log-convex functions as a special cases of log-s-convex fuzzy-IVF in the second sense.

\section{Remark 2.14}

(i) If $s=1$, then log-s-convex fuzzy-IVF in the second sense becomes log-convex fuzzy-IVF in the second sense, see [27], that is

$$
\mathcal{T}(\tau x+(1-\tau) y) \leqslant \mathcal{T}(x)^{\tau} \tilde{x} \mathcal{T}(y)^{1-\tau}, \quad \forall x, y \in K, \tau \in[0,1]
$$

(ii) If $s \equiv 0$, then $\log$-s-convex fuzzy-IVF in the second sense becomes $\log -P$-convex fuzzy-IVF, that is

$$
\mathcal{T}(\tau x+(1-\tau) y) \leqslant \mathcal{T}(x) \tilde{\times} \mathcal{T}(y), \quad \forall x, y \in K, \tau \in[0,1] .
$$

Theorem 2.15 Let $K$ be a convex set let $\mathcal{T}: K \rightarrow \mathbb{F}_{C}(\mathbb{R})$ be a fuzzy-IVF with $\mathcal{T}(x) \geqslant \tilde{0}$, whose $\alpha$-levels define the family of IVFs $\mathcal{T}_{\alpha}: K \subset \mathbb{R} \rightarrow \mathcal{K}_{C}^{+} \subset \mathcal{K}_{C}$ are given by

$\mathcal{T}_{\alpha}(x)=\left[\mathcal{T}_{*}(x, \alpha), \mathcal{T}^{*}(x, \alpha)\right]$,

for all $x \in K$ and for all $\alpha \in(0,1]$. Then $\mathcal{T}$ is log-s-convex in the second sense on $K$, if and only if, for all $\alpha \in(0,1]$, $\mathcal{T}_{*}(x, \alpha)$ and $\mathcal{T}^{*}(x, \alpha)$ both are log-s-convex in the second sense.
Proof Let $\mathcal{T}$ is $\log$-s-convex fuzzy-IVF in the second sense on $K$. Then for all $x, y \in K$ and $\tau \in[0,1]$, we have

$\mathcal{T}(\tau x+(1-\tau) y) \leqslant \mathcal{T}(x)^{\tau^{s}} \tilde{x} \mathcal{T}(y)^{(1-\tau)^{s}}$.

Therefore, from inequality (20) and Proposition 2.4, we have

$$
\begin{aligned}
& {\left[\mathcal{T}_{*}(\tau x+(1-\tau) y, \alpha), \mathcal{T}^{*}(\tau x+(1-\tau) y, \alpha)\right]} \\
& \quad \leq_{l}\left[\mathcal{T}_{*}(x, \alpha)^{\tau^{s}}, \mathcal{T}^{*}(x, \alpha)^{\tau^{s}}\right] \times\left[\mathcal{T}_{*}(y, \alpha)^{(1-\tau)^{s}}, \mathcal{T}^{*}(y, \alpha)^{(1-\tau)^{s}}\right] .
\end{aligned}
$$

It follows that

$\mathcal{T}_{*}(\tau x+(1-\tau) y, \alpha) \leq \mathcal{T}_{*}(x, \alpha)^{\tau^{s}} \mathcal{T}_{*}(y, \alpha)^{(1-\tau)^{s}}$,

and

$\mathcal{T}^{*}(\tau x+(1-\tau) y, \alpha) \leq \mathcal{T}^{*}(x, \alpha)^{\tau^{s}} \mathcal{T}^{*}(y, \alpha)^{(1-\tau)^{s}}$,

for each $\alpha \in(0,1]$. This shows that $\mathcal{T}_{*}(x, \alpha)$ and $\mathcal{T}^{*}(x, \alpha)$ both are $\log -s$-convex functions in the second sense.

Conversely, suppose that $\mathcal{T}_{*}(x, \alpha)$ and $\mathcal{T}^{*}(x, \alpha)$ both are $\log -s$-convex functions in the second sense. Then from definition and inequality (21), it follows that $\mathcal{T}(x)$ is log-s-convex fuzzy-IVF in the second sense.

Example 2.16 We consider the fuzzy-IVF $\mathcal{T}:[1,4] \rightarrow \mathbb{F}_{C}(\mathbb{R})$ defined by,

$\mathcal{T}(x)(\sigma)= \begin{cases}\frac{\sigma}{\frac{T}{x}} & \sigma \in\left[0, \frac{1}{x}\right] \\ \frac{\frac{2}{x}}{x} \sigma & \sigma \in\left(\mathrm{e}^{x^{2}}, 2 \mathrm{e}^{x^{2}}\right] \\ \frac{\frac{1}{x}}{\frac{1}{x}} & \text { otherwise. }\end{cases}$

Then, for each $\alpha \in(0,1]$, we have $\mathcal{T}_{\alpha}(x)=\left[\alpha \frac{1}{x},(2-\alpha) \frac{1}{x}\right]$. Since end point functions $\mathcal{T}_{*}(x, \alpha), \mathcal{T}^{*}(x, \alpha)$ are log-s-convex functions in the second sense for each $\alpha \in(0,1]$ then, by Theorem $2.15 \mathcal{T}(x)$ is log-s-convex fuzzy-IVF in the second sense.

Corollary 2.17 Let $K$ be a convex set let $\mathcal{T}: K \rightarrow \mathbb{F}_{C}(\mathbb{R})$ be a fuzzy-IVF with $\mathcal{T}(x) \geqslant 0$, whose $\alpha$-levels define the family of IVFs $\mathcal{T}_{\alpha}: K \subset \mathbb{R} \rightarrow \mathcal{K}_{C}^{+} \subset \mathcal{K}_{C}$ are given by

$\mathcal{T}_{\alpha}(x)=\left[\mathcal{T}_{*}(x, \alpha), \mathcal{T}^{*}(x, \alpha)\right]$,

for all $x \in K$ and for all $\alpha \in(0,1]$. Then $\mathcal{T}$ is log-convex in the second sense on $K$, if and only if, for all $\alpha \in(0,1]$, $\mathcal{T}_{*}(x, \alpha)$ and $\mathcal{T}^{*}(x, \alpha)$ both are log-convex in the second sense.

Corollary 2.18 Let $K$ be a convex set let $\mathcal{T}: K \rightarrow \mathbb{F}_{C}(\mathbb{R})$ be a fuzzy-IVF with $\mathcal{T}(x) \geqslant 0$, whose $\alpha$-levels define the family of IVFs $\mathcal{T}_{\alpha}: K \subset \mathbb{R} \rightarrow \mathcal{K}_{C}^{+} \subset \mathcal{K}_{C}$ are given by 
$\mathcal{T}_{\alpha}(x)=\left[\mathcal{T}_{*}(x, \alpha), \mathcal{T}^{*}(x, \alpha)\right]$,

for all $x \in K$ and for all $\alpha \in(0,1]$. Then $\mathcal{T}$ is log-P-convex on $K$, if and only if, for all $\alpha \in(0,1], \mathcal{T}_{*}(x, \alpha)$ and $\mathcal{T}^{*}(x, \alpha)$ both are log-P-convex.

\section{Remark 2.19}

(i) If $\mathcal{T}_{*}(u, \alpha)=\mathcal{T}^{*}(\vartheta, \alpha)$ with $\alpha=1$ then log-s-convex fuzzy-IVF in the second sense becomes log-s-convex function in the second sense, see [7].

(ii) If $\mathcal{T}_{*}(u, \alpha)=\mathcal{T}^{*}(\vartheta, \alpha)$ with $\alpha=1$ and $s=1$ then log-s -convex function becomes log-convex function, see [9].

(iii) If $\mathcal{T}_{*}(u, \alpha)=\mathcal{T}^{*}(\vartheta, \alpha)$ with $\alpha=1$ and $s=0$ then log-s -convex function reduces to the log- $P$-convex function, see [10].

Theorem 2.20 Let $K$ be convex set and $\mathcal{T}: K \rightarrow \mathbb{F}_{C}(\mathbb{R})$ be a fuzzy-IVF, whose $\alpha$-levels define the family of IVFs $\mathcal{T}_{\alpha}: K \subset \mathbb{R} \rightarrow \mathcal{K}_{C}^{+} \subset \mathcal{K}_{C}$ are given by

$\mathcal{T}_{\alpha}(x)=\left[\mathcal{T}_{*}(x, \alpha), \mathcal{T}^{*}(x, \alpha)\right]$

for all $x \in K$ and for all $\alpha \in(0,1]$. Then $\mathcal{T}$ is log-s-concave in the second sense on $K$, if and only if, for all $\alpha \in(0,1]$, $\mathcal{T}_{*}(x, \alpha)$ and $\mathcal{T}^{*}(x, \alpha)$ are log-s-concave functions in the second sense.

Proof Proof is similar to the proof of Theorem 2.15.

Example 2.21 We consider the fuzzy IVFs $\mathcal{T}:[u, \vartheta]=[0,8] \rightarrow \mathbb{F}_{C}(\mathbb{R})$ defined by,

$\mathcal{T}(x)(\sigma)= \begin{cases}\frac{\sigma}{x}, & \sigma \in[0, x], \\ \frac{2 x-\sigma}{x}, & \sigma \in(x, 2 x], \\ 0, & \text { otherwise. }\end{cases}$

Then, for each $\alpha \in(0,1]$, we have

$\mathcal{T}_{\alpha}(x)=[\alpha x,(2-\alpha) x]$.

Since end point functions $\mathcal{T}_{*}(x, \alpha)=\alpha x$, $\mathcal{T}^{*}(x, \alpha)=(2-\alpha) x$ are log-s-concave functions in the second sense, for each $\alpha \in(0,1]$ then, by Theorem $2.20 \mathcal{T}(x)$ is $\log$-s-concave fuzzy-IVF in the second sense.

\section{Main results}

This section presents Hermite-Hadamard, Hermite-Hadamard-Fejér and Jensen's inequalities for $\log$ - $s$-convex fuzzy-interval-valued functions in the second sense, and verify with the help of useful examples. First of all, we prove
Hermite-Hadamard inequalities for log-s-convex fuzzyinterval-valued functions.

Theorem 3.1 Let $\mathcal{T}:[u, \vartheta] \rightarrow \mathbb{F}_{C}(\mathbb{R})$ be a log-s-convex fuzzy-IVF in the second sense, whose $\alpha$-levels define the family of IVFs $\mathcal{T}_{\alpha}:[u, \vartheta] \subset \mathbb{R} \rightarrow \mathcal{K}_{C}^{+}$are given by $\mathcal{T}_{\alpha}(x)=\left[\mathcal{T}_{*}(x, \alpha), \mathcal{T}^{*}(x, \alpha)\right]$ for all $x \in[u, \vartheta]$ and for all $\alpha \in(0,1]$. If $\mathcal{T} \in \mathcal{F} \mathcal{R}_{([u, 9], \alpha)}$, then

$\mathcal{T}\left(\frac{u+\vartheta}{2}\right)^{2^{s-1}} \leqslant \exp \left[\frac{1}{\vartheta-u}(F R) \int_{u}^{\vartheta} \ln \mathcal{T}(x) \mathrm{d} x\right] \leqslant[\mathcal{T}(u) \tilde{\times} \mathcal{T}(\vartheta)]^{\frac{1}{s+1}}$.

Proof Let $\mathcal{T}:[u, \vartheta] \rightarrow \mathbb{F}_{C}(\mathbb{R}), \log$-s-convex fuzzy-IVF in the second sense. Then, by hypothesis, we have

$\mathcal{T}\left(\frac{u+\vartheta}{2}\right) \leqslant[\mathcal{T}(\tau u+(1-\tau) \vartheta)]^{\frac{1}{2^{s}}} \tilde{x}[\mathcal{T}((1-\tau) u+\tau \vartheta)]^{\frac{1}{2^{s}}}$.

Therefore, for every $\alpha \in(0,1]$, we have

$\mathcal{T}_{*}\left(\frac{u+\vartheta}{2}, \alpha\right) \leq\left[\mathcal{T}_{*}(\tau u+(1-\tau) \vartheta, \alpha)\right]^{\frac{1}{2^{s}}} \times\left[\mathcal{T}_{*}((1-\tau) u+\tau \vartheta, \alpha)\right]^{\frac{1}{2^{s}}}$, $\mathcal{T}^{*}\left(\frac{u+\vartheta}{2}, \alpha\right) \leq\left[\mathcal{T}^{*}(\tau u+(1-\tau) \vartheta, \alpha)\right]^{\frac{1}{2^{s}}} \times\left[\mathcal{T}^{*}((1-\tau) u+\tau \vartheta, \alpha)\right]^{\frac{1}{2^{s}}}$.

Taking logarithms on both sides of (25), then we obtain

$\frac{1}{\frac{1}{2^{s}}} \ln \mathcal{T}_{*}\left(\frac{u+\vartheta}{2}, \alpha\right) \leq \ln \mathcal{T}_{*}(\tau u+(1-\tau) \vartheta, \alpha)+\ln \mathcal{T}_{*}((1-\tau) u+\tau \vartheta, \alpha)$, $\frac{1}{\frac{1}{2^{s}}} \ln \mathcal{T}^{*}\left(\frac{u+\vartheta}{2}, \alpha\right) \leq \ln \mathcal{T}^{*}(\tau u+(1-\tau) \vartheta, \alpha)+\ln \mathcal{T}^{*}((1-\tau) u+\tau \vartheta, \alpha)$.

Then,

$\frac{1}{\frac{1}{2^{s}}} \int_{0}^{1} \ln \mathcal{T}_{*}\left(\frac{u+\vartheta}{2}, \alpha\right) \mathrm{d} \tau \leq \int_{0}^{1} \ln \mathcal{T}_{*}(\tau u+(1-\tau) \vartheta, \alpha) \mathrm{d} \tau$

$+\int_{0}^{1} \ln \mathcal{T}_{*}((1-\tau) u+\tau \vartheta, \alpha) \mathrm{d} \tau$,

$\frac{1}{\frac{1}{2^{s}}} \int_{0}^{1} \ln \mathcal{T}^{*}\left(\frac{u+\vartheta}{2}, \alpha\right) \mathrm{d} \tau \leq \int_{0}^{1} \ln \mathcal{T}^{*}(\tau u+(1-\tau) \vartheta, \alpha) \mathrm{d} \tau$

$+\int_{0}^{1} \ln \mathcal{T}^{*}((1-\tau) u+\tau \vartheta, \alpha) \mathrm{d} \tau$.

It follows that

$\frac{1}{2 \frac{1}{2 s}} \ln \mathcal{T}_{*}\left(\frac{u+\vartheta}{2}, \alpha\right) \leq \frac{1}{9-u} \int_{u}^{\vartheta} \ln \mathcal{T}_{*}(x, \alpha) \mathrm{d} x$,

$\frac{1}{2 \frac{1}{2^{s}}} \ln \mathcal{T}^{*}\left(\frac{u+\vartheta}{2}, \alpha\right) \leq \frac{1}{\vartheta-u} \int_{u}^{\vartheta} \ln \mathcal{T}^{*}(x, \alpha) \mathrm{d} x$,

which implies that

$\mathcal{T}_{*}\left(\frac{u+\vartheta}{2}, \alpha\right)^{2^{s-1}} \leq \exp \left(\frac{1}{\vartheta-u} \int_{u}^{\vartheta} \ln \mathcal{T}_{*}(x, \alpha) \mathrm{d} x\right)$,

$\mathcal{T}^{*}\left(\frac{u+\vartheta}{2}, \alpha\right)^{2^{s-1}} \leq \exp \left(\frac{1}{\vartheta-u} \int_{u}^{\vartheta} \ln \mathcal{T}^{*}(x, \alpha) \mathrm{d} x\right)$. 
That is

$\left[\mathcal{T}_{*}\left(\frac{u+\vartheta}{2}, \alpha\right)^{2^{s-1}}, \mathcal{T}^{*}\left(\frac{u+\vartheta}{2}, \alpha\right)^{2^{s-1}}\right] \leq_{I}\left[\exp \left(\frac{1}{\vartheta-u} \int_{u}^{\vartheta} \ln \mathcal{T}_{*}(x, \alpha) \mathrm{d} x\right), \exp \left(\frac{1}{\vartheta-u} \int_{u}^{\vartheta} \ln \mathcal{T}^{*}(x, \alpha) \mathrm{d} x\right)\right]$

Thus,

$\mathcal{T}\left(\frac{u+\vartheta}{2}\right)^{2^{s-1}} \preccurlyeq \exp \left[\frac{1}{\vartheta-u}(F R) \int_{u}^{\vartheta} \ln \mathcal{T}(x) \mathrm{d} x\right]$

In a similar way as above, we have

$\exp \left[\frac{1}{\vartheta-u}(F R) \int_{u}^{\vartheta} \ln \mathcal{T}(x) \mathrm{d} x\right] \preccurlyeq[\mathcal{T}(u) \tilde{\times} \mathcal{T}(\vartheta)]^{\frac{1}{s+1}}$

Combining (26) and (27), we have

$\mathcal{T}\left(\frac{u+\vartheta}{2}\right)^{2^{s-1}} \leqslant \exp \left[\frac{1}{\vartheta-u}(F R) \int_{u}^{\vartheta} \ln \mathcal{T}(x) \mathrm{d} x\right] \leqslant[\mathcal{T}(u) \tilde{\times} \mathcal{T}(\vartheta)]^{\frac{1}{s+1}}$

the required result.

Now we discuss some special cases of Theorem 3.1.

Note that the demonstration of proof of Corollary 3.2 is similar to proof of Corollary 3.3. If $s=1$ then, from Theorem 3.1, we obtain the following result.

Corollary 3.2 Let $\mathcal{T}:[u, \vartheta] \rightarrow \mathbb{F}_{C}(\mathbb{R})$ be a log-convex fuzzy-IVF in the second sense, whose $\alpha$-levels define the family of IVFs $\mathcal{T}_{\alpha}:[u, \vartheta] \subset \mathbb{R} \rightarrow \mathcal{K}_{C}^{+}$are given by $\mathcal{T}_{\alpha}(x)=\left[\mathcal{T}_{*}(x, \alpha), \mathcal{T}^{*}(x, \alpha)\right]$ for all $x \in[u, \vartheta]$ and for all $\alpha \in(0,1]$. If $\mathcal{T} \in \mathcal{F R}_{([u, \vartheta], \alpha)}$, then

$\mathcal{T}\left(\frac{u+\vartheta}{2}\right) \preccurlyeq \exp \left[\frac{1}{\vartheta-u}(F R) \int_{u}^{\vartheta} \ln \mathcal{T}(x) \mathrm{d} x\right] \leqslant[\mathcal{T}(u) \tilde{\times} \mathcal{T}(\vartheta)]^{\frac{1}{2}}$.

If $s=0$ then, from Theorem 3.1, we obtain the following result for log- $P$-convex fuzzy-IVF.

Corollary 3.3 Let $\mathcal{T}:[u, \vartheta] \rightarrow \mathbb{F}_{C}(\mathbb{R})$ be a log-P-convex fuzzy-IVF, whose $\alpha$-levels define the family of IVFs $\mathcal{T}_{\alpha}:[u, \vartheta] \subset \mathbb{R} \rightarrow \mathcal{K}_{C}^{+}$are given by $\mathcal{T}_{\alpha}(x)=\left[\mathcal{T}_{*}(x, \alpha), \mathcal{T}^{*}(x, \alpha)\right]$ for all $x \in[u, \vartheta]$ and for all $\alpha \in(0,1]$. If $\mathcal{T} \in \mathcal{F} \mathcal{R}_{([u, \vartheta], \alpha)}$, then

$\mathcal{T}\left(\frac{u+\vartheta}{2}\right)^{\frac{1}{2}} \preccurlyeq \exp \left[\frac{1}{\vartheta-u}(F R) \int_{u}^{\vartheta} \ln \mathcal{T}(x) \mathrm{d} x\right] \preccurlyeq \mathcal{T}(u) \tilde{\times} \mathcal{T}(\vartheta)$.

Proof Let $\mathcal{T}:[u, \vartheta] \rightarrow \mathbb{F}_{C}(\mathbb{R}), \log -P$-convex fuzzy-IVF. Then, by hypothesis, we have

$\mathcal{T}\left(\frac{u+\vartheta}{2}\right) \leqslant \mathcal{T}(\tau u+(1-\tau) \vartheta) \tilde{\times} \mathcal{T}((1-\tau) u+\tau \vartheta)$.

Therefore, for every $\alpha \in(0,1]$, we have
$\mathcal{T}_{*}\left(\frac{u+\vartheta}{2}, \alpha\right) \leq \mathcal{T}_{*}(\tau u+(1-\tau) \vartheta, \alpha) \times \mathcal{T}_{*}((1-\tau) u+\tau \vartheta, \alpha)$, $\mathcal{T}^{*}\left(\frac{u+\vartheta}{2}, \alpha\right) \leq \mathcal{T}^{*}(\tau u+(1-\tau) \vartheta, \alpha) \times \mathcal{T}^{*}((1-\tau) u+\tau \vartheta, \alpha)$.

Taking logarithms on both sides of (29), then we obtain $\ln \mathcal{T}_{*}\left(\frac{u+\vartheta}{2}, \alpha\right) \leq \ln \mathcal{T}_{*}(\tau u+(1-\tau) \vartheta, \alpha)+\ln \mathcal{T}_{*}((1-\tau) u+\tau \vartheta, \alpha)$, $\ln \mathcal{T}^{*}\left(\frac{u+\vartheta}{2}, \alpha\right) \leq \ln \mathcal{T}^{*}(\tau u+(1-\tau) \vartheta, \alpha)+\ln \mathcal{T}^{*}((1-\tau) u+\tau \vartheta, \alpha)$.

Then,

$\int_{0}^{1} \ln \mathcal{T}_{*}\left(\frac{u+\vartheta}{2}, \alpha\right) \mathrm{d} \tau \leq \int_{0}^{1} \ln \mathcal{T}_{*}(\tau u+(1-\tau) \vartheta, \alpha) \mathrm{d} \tau$

$+\int_{0}^{1} \ln \mathcal{T}_{*}((1-\tau) u+\tau \vartheta, \alpha) \mathrm{d} \tau$,

$\int_{0}^{1} \ln \mathcal{T}^{*}\left(\frac{u+\vartheta}{2}, \alpha\right) \mathrm{d} \tau \leq \int_{0}^{1} \ln \mathcal{T}^{*}(\tau u+(1-\tau) \vartheta, \alpha) \mathrm{d} \tau$

$+\int_{0}^{1} \ln \mathcal{T}^{*}((1-\tau) u+\tau \vartheta, \alpha) \mathrm{d} \tau$.

It follows that

$\frac{1}{2} \ln \mathcal{T}_{*}\left(\frac{u+\vartheta}{2}, \alpha\right) \leq \frac{1}{\vartheta-u} \int_{u}^{\vartheta} \ln \mathcal{T}_{*}(x, \alpha) \mathrm{d} x$,

$\frac{1}{2} \ln \mathcal{T}^{*}\left(\frac{u+\vartheta}{2}, \alpha\right) \leq \frac{1}{\vartheta-u} \int_{u}^{\vartheta} \ln \mathcal{T}^{*}(x, \alpha) \mathrm{d} x$,

which implies that

$\left[\mathcal{T}_{*}\left(\frac{u+\vartheta}{2}, \alpha\right)\right]^{\frac{1}{2}} \leq \exp \left(\frac{1}{\vartheta-u} \int_{u}^{\vartheta} \ln \mathcal{T}_{*}(x, \alpha) \mathrm{d} x\right)$,

$\left[\mathcal{T}^{*}\left(\frac{u+\vartheta}{2}, \alpha\right)\right]^{\frac{1}{2}} \leq \exp \left(\frac{1}{\vartheta-u} \int_{u}^{\vartheta} \ln \mathcal{T}^{*}(x, \alpha) \mathrm{d} x\right)$,

that is

$\left[\left[\mathcal{T}_{*}\left(\frac{u+\vartheta}{2}, \alpha\right)\right]^{\frac{1}{2}},\left[\mathcal{T}^{*}\left(\frac{u+\vartheta}{2}, \alpha\right)\right]^{\frac{1}{2}}\right]$
$\quad \leq_{I}\left[\exp \left(\frac{1}{\vartheta-u} \int_{u}^{\vartheta} \ln \mathcal{T}_{*}(x, \alpha) \mathrm{d} x\right), \exp \left(\frac{1}{\vartheta-u} \int_{u}^{\vartheta} \ln \mathcal{T}^{*}(x, \alpha) \mathrm{d} x\right)\right]$.

Thus,

$\mathcal{T}\left(\frac{u+\vartheta}{2}\right)^{\frac{1}{2}} \preccurlyeq \exp \left[\frac{1}{\vartheta-u}(F R) \int_{u}^{\vartheta} \ln \mathcal{T}(x) \mathrm{d} x\right]$.

In a similar way as above, we have

$\exp \left[\frac{1}{\vartheta-u}(F R) \int_{u}^{\vartheta} \ln \mathcal{T}(x) \mathrm{d} x\right] \preccurlyeq \mathcal{T}(u) \tilde{\times} \mathcal{T}(\vartheta)$

Combining (30) and (31), we have 
$\mathcal{T}\left(\frac{u+\vartheta}{2}\right)^{\frac{1}{2}} \preccurlyeq \exp \left[\frac{1}{\vartheta-u}(F R) \int_{u}^{\vartheta} \ln \mathcal{T}(x) \mathrm{d} x\right] \preccurlyeq \mathcal{T}(u) \tilde{\times} \mathcal{T}(\vartheta)$.

the required result.

Remark 3.4 If $\mathcal{T}_{*}(u, \alpha)=\mathcal{T}^{*}(\vartheta, \alpha)$ with $\alpha=1$, then Theorem 3.1 reduces to the result for log-s-convex fuzzy-IVF in the second sense, see [10]:

$\mathcal{T}\left(\frac{u+\vartheta}{2}\right)^{2^{s-1}} \leq \exp \left[\frac{1}{\vartheta-u}(R) \int_{u}^{\vartheta} \ln \mathcal{T}(x) \mathrm{d} x\right] \leq[\mathcal{T}(u) \times \mathcal{T}(\vartheta)]^{\frac{1}{s+1}}$.

If $\mathcal{T}_{*}(u, \alpha)=\mathcal{T}^{*}(\vartheta, \alpha)$ with $\alpha=1$ and $s=1$, then Theorem 3.1 reduces to the result for log-convex function see [23]:

$\mathcal{T}\left(\frac{u+\vartheta}{2}\right) \leq \exp \left[\frac{1}{\vartheta-u}(R) \int_{u}^{\vartheta} \ln \mathcal{T}(x) \mathrm{d} x\right] \leq \sqrt{\mathcal{T}(u) \times \mathcal{T}(\vartheta)}$.

If $\mathcal{T}_{*}(u, \alpha)=\mathcal{T}^{*}(\vartheta, \alpha)$ with $\alpha=1$ and $s=0$ then Theorem 3.1 reduces to the result for $\log -P$-convex function, see [10]:

$\mathcal{T}\left(\frac{u+\vartheta}{2}\right)^{\frac{1}{2}} \leq \exp \left[\frac{1}{\vartheta-u}(R) \int_{u}^{\vartheta} \ln \mathcal{T}(x) \mathrm{d} x\right] \leq \mathcal{T}(u) \times \mathcal{T}(\vartheta)$.

Example 3.5 We consider the fuzzy-IVF $\mathcal{T}:[u, \vartheta]=[1,4] \rightarrow \mathbb{F}_{C}(\mathbb{R}) \quad$ d e f i n e d $\quad$ b y , $\mathcal{T}_{\alpha}(x)=\left[\alpha \mathrm{e}^{x^{2}},(2-\alpha) \mathrm{e}^{x^{2}}\right]$, then $\mathcal{T}(x)$ is log-s-convex fuzzy-IVF in the second sense. Since, $\mathcal{T}_{*}(x, \alpha)=\alpha \mathrm{e}^{x^{2}}$ and $\mathcal{T}^{*}(x, \alpha)=(2-\alpha) \mathrm{e}^{x^{2}}$ then, we have

$$
\begin{aligned}
& \mathcal{T}_{*}\left(\frac{u+\vartheta}{2}, \alpha\right)^{2^{s-1}}=\alpha \mathrm{e}^{\left(\frac{5}{2}\right)^{2}}=\alpha \mathrm{e}^{\frac{25}{4}}, \\
& \exp \left(\frac{1}{\vartheta-u} \int_{u}^{\vartheta} \ln \mathcal{T}_{*}(x, \alpha) \mathrm{d} x\right) \\
& \quad=\exp \left(\frac{1}{3} \int_{1}^{4} \ln \left(\alpha \mathrm{e}^{x^{2}}\right) \mathrm{d} x\right)=\mathrm{e}^{\ln (\alpha)+7},
\end{aligned}
$$

$\left[\mathcal{T}_{*}(u) \times \mathcal{T}_{*}(\vartheta)\right]^{\frac{1}{s+1}}=\left[(\alpha e)\left(4 \alpha \mathrm{e}^{16}\right)\right]^{\frac{1}{2}}=2 \alpha \mathrm{e}^{\frac{17}{2}}$,

for all $\alpha \in(0,1]$. That means

$\alpha \mathrm{e}^{\frac{25}{4}} \leq \mathrm{e}^{\ln (\alpha)+7} \leq 2 \alpha \mathrm{e}^{\frac{17}{2}}$.

Similarly, it can be easily show that

$$
\begin{aligned}
& \mathcal{T}^{*}\left(\frac{u+\vartheta}{2}, \alpha\right)^{2^{s-1}} \leq \exp \left[\frac{1}{\vartheta-u} \int_{u}^{\vartheta} \ln \mathcal{T}^{*}(x, \alpha) \mathrm{d} x\right] \\
& \quad \leq\left[\mathcal{T}^{*}(u, \alpha) \times \mathcal{T}^{*}(\vartheta, \alpha)\right]^{\frac{1}{s+1}} .
\end{aligned}
$$

for all $\alpha \in(0,1]$, such that
$\mathcal{T}^{*}\left(\frac{u+\vartheta}{2}, \alpha\right)^{2^{s-1}}=(2-\alpha) \mathrm{e}^{\left(\frac{5}{2}\right)^{2}}=(2-\alpha) \mathrm{e}^{\frac{25}{4}}$,

$$
\begin{aligned}
& \exp \left(\frac{1}{\vartheta-u} \int_{u}^{\vartheta} \ln \mathcal{T}^{*}(x, \alpha) \mathrm{d} x\right) \\
& \quad=\exp \left(\frac{1}{3} \int_{1}^{4} \ln \left((2-\alpha) \mathrm{e}^{x^{2}}\right) \mathrm{d} x\right)=\mathrm{e}^{\ln (2-\alpha)+7},
\end{aligned}
$$

$\left[\mathcal{T}^{*}(u, \alpha) \times \mathcal{T}^{*}(\vartheta, \alpha)\right]^{\frac{1}{s+1}}=\left[(2-\alpha) e \cdot 4(2-\alpha) \mathrm{e}^{16}\right]^{\frac{1}{2}}=2(2-\alpha) \mathrm{e}^{\frac{17}{2}}$.

From which, it follows that

$(2-\alpha) \mathrm{e}^{\frac{25}{4}} \leq \mathrm{e}^{\ln (2-\alpha)+7} \leq 2(2-\alpha) \mathrm{e}^{\frac{17}{2}}$,

that is

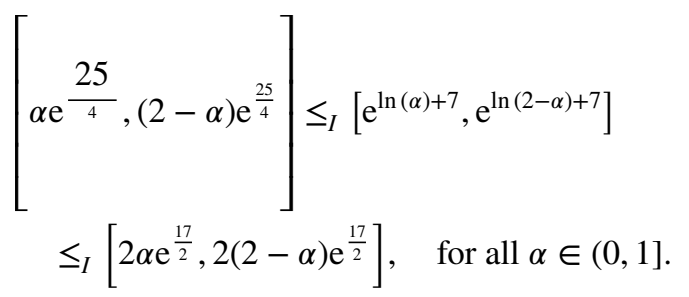

Hence,

$\mathcal{T}\left(\frac{u+\vartheta}{2}\right)^{2^{s-1}} \preccurlyeq \exp \left[\frac{1}{\vartheta-u}(F R) \int_{u}^{\vartheta} \ln \mathcal{T}(x) \mathrm{d} x\right] \leqslant[\mathcal{T}(u) \tilde{\times} \mathcal{T}(\vartheta)]^{\frac{1}{s+1}}$.

As we know that $\mathrm{HH}-$ Fejér inequality is generalized $\mathrm{HH}-$ Fejér inequality and with the help of this inequality, we can derive some special inequalities like $\mathrm{HH}$-inequality. Firstly, we obtain the second $\mathrm{HH}-$ Fejér inequality for log$s$-convex fuzzy-IVF in the second sense.

Theorem 3.6 Let $\mathcal{T}:[u, \vartheta] \rightarrow \mathbb{F}_{C}(\mathbb{R})$ be a log-s-convex fuzzy-IVF in the second sense with $u<\vartheta$, whose $\alpha$-levels define the family of IVFs $\mathcal{T}_{\alpha}:[u, \vartheta] \subset \mathbb{R} \rightarrow \mathcal{K}_{C}^{+}$are given by $\mathcal{T}_{\alpha}(x)=\left[\mathcal{T}_{*}(x, \alpha), \mathcal{T}^{*}(x, \alpha)\right]$ for all $x \in[u, \vartheta]$ and for all $\alpha \in(0,1]$. If $\mathcal{T} \in \mathcal{F R}_{([u, \vartheta], \alpha)}$ and $\Omega:[u, \vartheta] \rightarrow \mathbb{R}, \Omega(x) \geq 0$, symmetric with respect to $\frac{u+\vartheta}{2}$, then

$\frac{1}{\vartheta-u}(F R) \int_{u}^{\vartheta}[\ln \mathcal{T}(x)] \Omega(x) \mathrm{d} x \leqslant \ln [\mathcal{T}(u) \tilde{\times} \mathcal{T}(\vartheta)] \int_{0}^{1} \tau^{s} \Omega((1-\tau) u+\tau \vartheta) \mathrm{d} \tau$.

Proof Let $\mathcal{T}$ be a $\log$-s-convex fuzzy-IVF in the second sense. Then, for each $\alpha \in(0,1]$, we have

$$
\begin{aligned}
& \quad\left[\ln \mathcal{T}_{*}(\tau u+(1-\tau) \vartheta, \alpha)\right] \Omega(\tau u+(1-\tau) \vartheta) \\
& \quad \leq\left(\tau^{s} \ln \mathcal{T}_{*}(u, \alpha)+(1-\tau)^{s} \ln \mathcal{T}_{*}(\vartheta, \alpha)\right) \Omega(\tau u+(1-\tau) \vartheta), \\
& {\left[\ln \mathcal{T}^{*}(\tau u+(1-\tau) \vartheta, \alpha)\right] \Omega(\tau u+(1-\tau) \vartheta)} \\
& \quad \leq\left(\tau^{s} \ln \mathcal{T}^{*}(u, \alpha)+(1-\tau)^{s} \ln \mathcal{T}^{*}(\vartheta, \alpha)\right) \Omega(\tau u+(1-\tau) \vartheta) .
\end{aligned}
$$


And

$\left[\ln \mathcal{T}_{*}((1-\tau) u+\tau \vartheta, \alpha)\right] \Omega((1-\tau) u+\tau \vartheta)$

$\leq\left((1-\tau)^{s} \ln \mathcal{T}_{*}(u, \alpha)+\tau^{s} \ln \mathcal{T}_{*}(\vartheta, \alpha)\right) \Omega((1-\tau) u+\tau \vartheta)$,

$\left[\ln \mathcal{T}^{*}((1-\tau) u+\tau \vartheta, \alpha)\right] \Omega((1-\tau) u+\tau \vartheta)$

$\leq\left((1-\tau)^{s} \ln \mathcal{T}^{*}(u, \alpha)+\tau^{s} \ln \mathcal{T}^{*}(\vartheta, \alpha)\right) \Omega((1-\tau) u+\tau \vartheta)$.

After adding (33) and (34), and integrating over [0, 1], we get that is

$\left[\frac{1}{\vartheta-u} \int_{u}^{\vartheta}\left[\ln \mathcal{T}_{*}(x, \alpha)\right] \Omega(x) \mathrm{d} x, \frac{1}{\vartheta-u} \int_{u}^{\vartheta}\left[\ln \mathcal{T}^{*}(x, \alpha)\right] \Omega(x) \mathrm{d} x\right]$

$\leq_{l}\left[\ln \left[T_{*}(u, \alpha) \times T_{*}(\vartheta, \alpha)\right], \ln \left[T^{*}(u, \alpha) \times T^{*}(\vartheta, \alpha)\right]\right] \int_{0}^{1} \tau^{s} \Omega((1-\tau) u+\tau \vartheta) \mathrm{d} \tau$,

hence

$$
\begin{aligned}
& \int_{0}^{1}\left[\ln \mathcal{T}_{*}(\tau u+(1-\tau) \vartheta, \alpha)\right] \Omega(\tau u+(1-\tau) \vartheta) \mathrm{d} \tau+\int_{0}^{1} \ln \mathcal{T}_{*}((1-\tau) u+\tau \vartheta, \alpha) \Omega((1-\tau) u+\tau \vartheta) \mathrm{d} \tau \\
& \quad \leq \int_{0}^{1}\left[\begin{array}{c}
\ln \mathcal{T}_{*}(u, \alpha)\left\{\tau^{s} \Omega(\tau u+(1-\tau) \vartheta)+(1-\tau)^{s} \Omega((1-\tau) u+\tau \vartheta)\right\} \\
+\ln \mathcal{T}_{*}(\vartheta, \alpha)\left\{(1-\tau)^{s} \Omega(\tau u+(1-\tau) \vartheta)+\tau^{s} \Omega((1-\tau) u+\tau \vartheta)\right\}
\end{array}\right] \mathrm{d} \tau, \\
& \int_{0}^{1}\left[\ln \mathcal{T}^{*}((1-\tau) u+\tau \vartheta, \alpha)\right] \Omega((1-\tau) u+\tau \vartheta) \mathrm{d} \tau+\int_{0}^{1} \ln \mathcal{T}^{*}(\tau u+(1-\tau) \vartheta, \alpha) \Omega(\tau u+(1-\tau) \vartheta) \mathrm{d} \tau \\
& \leq \int_{0}^{1}\left[\begin{array}{c}
\ln \mathcal{T}^{*}(u, \alpha)\left\{\tau^{s} \Omega(\tau u+(1-\tau) \vartheta)+(1-\tau)^{s} \Omega((1-\tau) u+\tau \vartheta)\right\} \\
+\ln \mathcal{T}^{*}(\vartheta, \alpha)\left\{(1-\tau)^{s} \Omega(\tau u+(1-\tau) \vartheta)+\tau^{s} \Omega((1-\tau) u+\tau \vartheta)\right\}
\end{array}\right] \mathrm{d} \tau .
\end{aligned}
$$$$
=2 \ln \mathcal{T}_{*}(u, \alpha) \int_{0}^{1} \tau^{s} \Omega(\tau u+(1-\tau) \vartheta) \mathrm{d} \tau+2 \ln \mathcal{T}_{*}(\vartheta, \alpha) \int_{0}^{1} \tau^{s} \Omega((1-\tau) u+\tau \vartheta) \mathrm{d} \tau,
$$$$
=2 \ln \mathcal{T}^{*}(u, \alpha) \int_{0}^{1} \tau^{s} \Omega(\tau u+(1-\tau) \vartheta) \mathrm{d} \tau+2 \ln \mathcal{T}^{*}(\vartheta, \alpha) \int_{0}^{1} \tau^{s} \Omega((1-\tau) u+\tau \vartheta) \mathrm{d} \tau \text {. }
$$

Since $\Omega$ is symmetric, then

$=2 \ln \left[\mathcal{T}_{*}(u, \alpha) \times \mathcal{T}_{*}(\vartheta, \alpha)\right] \int_{0}^{1} \tau^{s} \Omega((1-\tau) u+\tau \vartheta) \mathrm{d} \tau$,
$=2 \ln \left[\mathcal{T}^{*}(u, \alpha) \times \mathcal{T}^{*}(\vartheta, \alpha)\right] \int_{0}^{1} \tau^{s} \Omega((1-\tau) u+\tau \vartheta) \mathrm{d} \tau$

Since
$\frac{1}{\vartheta-u}(F R) \int_{u}^{\vartheta}[\ln \mathcal{T}(x)] \Omega(x) \mathrm{d} x \leqslant \ln [\mathcal{T}(u) \tilde{\times} \mathcal{T}(\vartheta)] \int_{0}^{1} \tau^{s} \Omega((1-\tau) u+\tau \vartheta) \mathrm{d} \tau$.

This concludes the proof.

Next, we construct first HH-Fejér inequality for log-s -convex fuzzy-IVF in the second sense, which generalizes first $\mathrm{HH}-\mathrm{Fejér}$ inequality for $\log -s$-convex function in the second sense.

$$
\begin{aligned}
& \int_{0}^{1}\left[\ln \mathcal{T}_{*}(\tau u+(1-\tau) \vartheta, \alpha)\right] \Omega(\tau u+(1-\tau) \vartheta) \mathrm{d} \tau \\
& \quad=\int_{0}^{1}\left[\ln \mathcal{T}_{*}((1-\tau) u+\tau \vartheta, \alpha)\right] \Omega((1-\tau) u+\tau \vartheta) \mathrm{d} \tau=\frac{1}{\vartheta-u} \int_{u}^{\vartheta}\left[\ln \mathcal{T}_{*}(x, \alpha)\right] \Omega(x) \mathrm{d} x \\
& \int_{0}^{1}\left[\ln \mathcal{T}^{*}((1-\tau) u+\tau \vartheta, \alpha)\right] \Omega((1-\tau) u+\tau \vartheta) \mathrm{d} \tau \\
& \quad=\int_{0}^{1}\left[\ln \mathcal{T}^{*}(\tau u+(1-\tau) \vartheta, \alpha)\right] \Omega(\tau u+(1-\tau) \vartheta) \mathrm{d} \tau=\frac{1}{\vartheta-u} \int_{u}^{\vartheta}\left[\ln \mathcal{T}^{*}(x, \alpha)\right] \Omega(x) \mathrm{d} x .
\end{aligned}
$$

From (35) and (36), we have

$$
\begin{aligned}
& \frac{1}{\vartheta-u} \int_{u}^{\vartheta}\left[\ln \mathcal{T}_{*}(x, \alpha)\right] \Omega(x) \mathrm{d} x \leq \ln \left[\mathcal{T}_{*}(u, \alpha) \times \mathcal{T}_{*}(\vartheta, \alpha)\right] \int_{0}^{1} \tau^{s} \Omega((1-\tau) u+\tau \vartheta) \mathrm{d} \tau, \\
& \frac{1}{\vartheta-u} \int_{u}^{\vartheta}\left[\ln \mathcal{T}^{*}(x, \alpha)\right] \Omega(x) \mathrm{d} x \leq \ln \left[\mathcal{T}^{*}(u, \alpha) \times \mathcal{T}^{*}(\vartheta, \alpha)\right] \int_{0}^{1} \tau^{s} \Omega((1-\tau) u+\tau \vartheta) \mathrm{d} \tau,
\end{aligned}
$$


Theorem 3.7 Let $\mathcal{T}:[u, \vartheta] \rightarrow \mathbb{F}_{C}(\mathbb{R})$ be a log-s-convex fuzzy-IVF in the second sense with $u<\vartheta$, whose $\alpha$-levels define the family of IVFs $\mathcal{T}_{\alpha}:[u, \vartheta] \subset \mathbb{R} \rightarrow \mathcal{K}_{C}^{+}$are given by $\mathcal{T}_{\alpha}(x)=\left[\mathcal{T}_{*}(x, \alpha), \mathcal{T}^{*}(x, \alpha)\right]$ for all $x \in[u, \vartheta]$ and for all $\alpha \in(0,1]$. If $\mathcal{T} \in \mathcal{F R}_{([u, \vartheta], \alpha)}$ and $\Omega:[u, \vartheta] \rightarrow \mathbb{R}, \Omega(x) \geq 0$, symmetric with respect to $\frac{u+\vartheta}{2}$, and $\int_{u}^{\vartheta} \Omega(x) \mathrm{d} x>0$, then

$\ln \mathcal{T}\left(\frac{u+\vartheta}{2}\right) \leqslant \frac{2^{1-s}}{\int_{u}^{\vartheta} \Omega(x) \mathrm{d} x}(F R) \int_{u}^{\vartheta}[\ln \mathcal{T}(x)] \Omega(x) \mathrm{d} x$.

Proof Since $\mathcal{T}$ is a log-s-convex in the second sense, then for $\alpha \in(0,1]$ we have

$2^{s} \ln \mathcal{T}_{*}\left(\frac{u+\vartheta}{2}, \alpha\right) \leq \ln \mathcal{T}_{*}(\tau u+(1-\tau) \vartheta, \alpha)+\ln \mathcal{T}_{*}((1-\tau) u+\tau \vartheta, \alpha)$, $2^{s} \ln \mathcal{T}^{*}\left(\frac{u+\vartheta}{2}, \alpha\right) \leq \ln \mathcal{T}^{*}(\tau u+(1-\tau) \vartheta, \alpha)+\ln \mathcal{T}^{*}((1-\tau) u+\tau \vartheta, \alpha)$.

B y multiplying (3 8 ) by $\Omega((1-\tau) u+\tau \vartheta)=\Omega(\tau u+(1-\tau) \vartheta)$ and integrate it by $\tau$ over $[0,1]$, we obtain

$$
\begin{aligned}
& 2^{s}[\left.\ln \mathcal{T}_{*}\left(\frac{u+\vartheta}{2}, \alpha\right)\right] \int_{0}^{1} \Omega((1-\tau) u+\tau \vartheta) \mathrm{d} \tau \\
& \leq \int_{0}^{1}\left[\ln \mathcal{T}_{*}(\tau u+(1-\tau) \vartheta, \alpha)\right] \Omega(\tau u+(1-\tau) \vartheta) \mathrm{d} \tau \\
& \quad+\int_{0}^{1}\left[\ln \mathcal{T}_{*}((1-\tau) u+\tau \vartheta, \alpha)\right] \Omega((1-\tau) u+\tau \vartheta) \mathrm{d} \tau, \\
& 2^{s}\left[\ln \mathcal{T}^{*}\left(\frac{u+\vartheta}{2}, \alpha\right)\right] \int_{0}^{1} \Omega((1-\tau) u+\tau \vartheta) \mathrm{d} \tau \\
& \leq \int_{0}^{1}\left[\ln \mathcal{T}^{*}(\tau u+(1-\tau) \vartheta, \alpha)\right] \Omega(\tau u+(1-\tau) \vartheta) \mathrm{d} \tau \\
&+\int_{0}^{1}\left[\ln \mathcal{T}^{*}((1-\tau) u+\tau \vartheta, \alpha)\right] \Omega((1-\tau) u+\tau \vartheta) \mathrm{d} \tau .
\end{aligned}
$$

Since

$$
\begin{aligned}
& \int_{0}^{1}\left[\ln \mathcal{T}_{*}(\tau u+(1-\tau) \vartheta, \alpha)\right] \Omega(\tau u+(1-\tau) \vartheta) \mathrm{d} \tau \\
& =\int_{0}^{1}\left[\ln \mathcal{T}_{*}((1-\tau) u+\tau \vartheta, \alpha)\right] \Omega((1-\tau) u+\tau \vartheta) \mathrm{d} \tau, \\
& =\frac{1}{\vartheta-u} \int_{u}^{\vartheta}\left[\ln \mathcal{T}_{*}(x, \alpha)\right] \Omega(x) \mathrm{d} x, \\
& \int_{0}^{1}\left[\ln \mathcal{T}^{*}(\tau u+(1-\tau) \vartheta, \alpha)\right] \Omega(\tau u+(1-\tau) \vartheta) \mathrm{d} \tau \\
& =\int_{0}^{1}\left[\ln \mathcal{T}^{*}((1-\tau) u+\tau \vartheta, \alpha)\right] \Omega((1-\tau) u+\tau \vartheta) \mathrm{d} \tau, \\
& =\frac{1}{\vartheta-u} \int_{u}^{\vartheta}\left[\ln \mathcal{T}^{*}(x, \alpha)\right] \Omega(x) \mathrm{d} x .
\end{aligned}
$$

$\ln \mathcal{T}_{*}\left(\frac{u+\vartheta}{2}, \alpha\right) \leq \frac{2^{1-s}}{\int_{u}^{\vartheta} \Omega(x) \mathrm{d} x} \int_{u}^{\vartheta}\left[\ln \mathcal{T}_{*}(x, \alpha)\right] \Omega(x) \mathrm{d} x$,

$\ln \mathcal{T}^{*}\left(\frac{u+\vartheta}{2}, \alpha\right) \leq \frac{2^{1-s}}{\int_{u}^{\vartheta} \Omega(x) \mathrm{d} x} \int_{u}^{\vartheta}\left[\ln \mathcal{T}^{*}(x, \alpha)\right] \Omega(x) \mathrm{d} x$.

From which, we have

$$
\begin{aligned}
& {\left[\ln \mathcal{T}_{*}\left(\frac{u+\vartheta}{2}, \alpha\right), \ln \mathcal{T}^{*}\left(\frac{u+\vartheta}{2}, \alpha\right)\right]} \\
& \quad \leq_{l} \frac{2^{1-s}}{\int_{u}^{\vartheta} \Omega(x) \mathrm{d} x}\left[\int_{u}^{\vartheta}\left[\ln \mathcal{T}_{*}(x, \alpha)\right] \Omega(x) \mathrm{d} x, \int_{u}^{\vartheta}\left[\ln \mathcal{T}^{*}(x, \alpha)\right] \Omega(x) \mathrm{d} x\right],
\end{aligned}
$$

that is

$\ln \mathcal{T}\left(\frac{u+\vartheta}{2}\right) \preccurlyeq \frac{2^{1-s}}{\int_{u}^{\vartheta} \Omega(x) \mathrm{d} x}(F R) \int_{u}^{\vartheta}[\ln \mathcal{T}(x)] \Omega(x) \mathrm{d} x$.

Then we complete the proof.

\section{Remark 3.8}

(i) If $s=1$ then inequalities in Theorems 3.6 and 3.7 reduces for log-convex fuzzy-IVFs.

(ii) If $\mathcal{T}_{*}(u, \alpha)=\mathcal{T}^{*}(u, \alpha)$ with $\alpha=1$, then Theorems 3.6 and 3.7 reduces to classical first and second $\mathrm{HH}-$ Fejér inequality for log-s-convex function in the second sense, see [19].

(iii) If $\mathcal{T}_{*}(u, \alpha)=\mathcal{T}^{*}(u, \alpha)$ with $\alpha=1$ and $s=1$ then Theorems 3.6 and 3.7 reduces to classical first and second $\mathrm{HH}-$ Fejér inequality for log-convex function, see [19].

Example 3.9 We consider $s=1$ for $\tau \in[0,1]$ and the fuzzy IVFs $\mathcal{T}:[u, \vartheta]=\left[\frac{\pi}{4}, \frac{\pi}{2}\right] \rightarrow \mathbb{F}_{C}(\mathbb{R})$ defined by,

$\mathcal{T}(x)(\sigma)= \begin{cases}\frac{\sigma}{\mathrm{e}^{\sin x},}, & \sigma \in\left[0, \mathrm{e}^{\sin x}\right], \\ \frac{2 \mathrm{e}^{\sin x}-\sigma}{\mathrm{e}^{\sin x}}, & \sigma \in\left(\mathrm{e}^{\sin x}, 2 \mathrm{e}^{\sin x}\right], \\ 0, & \text { otherwise. }\end{cases}$

Then, for each $\alpha \in(0,1]$, we have

$\mathcal{T}_{\alpha}(x)=\left[\alpha \mathrm{e}^{\sin x},(2-\alpha) \mathrm{e}^{\sin x}\right]$.

Since end point functions $\mathcal{T}_{*}(x, \alpha)=\alpha \mathrm{e}^{\sin x}$, $\mathcal{T}^{*}(x, \alpha)=(2-\alpha) \mathrm{e}^{\sin x}$ are log-1-convex functions in the second sense, for each $\alpha \in(0,1]$ then, by Theorem $2.15, \mathcal{T}(x)$ is $\log$-s-convex fuzzy-IVF in the second sense. If

$\Omega(x)=\left\{\begin{array}{l}x-\frac{\pi}{4}, \sigma \in\left[\frac{\pi}{4}, \frac{3 \pi}{8}\right], \\ \frac{\pi}{2}-x, \sigma \in\left(\frac{3 \pi}{8}, \frac{\pi}{2}\right],\end{array}\right.$

then, we have

From (39) and (40), we have 
$\frac{1}{\vartheta-u} \int_{u}^{\vartheta}\left[\ln \mathcal{T}_{*}(x, \alpha)\right] \Omega(x) \mathrm{d} x=\frac{4}{\pi} \int_{\frac{\pi}{4}}^{\frac{\pi}{2}}\left[\ln \mathcal{T}_{*}(x, \alpha)\right] \Omega(x) \mathrm{d} x=\frac{4}{\pi} \int_{\frac{\pi}{4}}^{\frac{3 \pi}{8}}\left[\ln \mathcal{T}_{*}(x, \alpha)\right] \Omega(x) \mathrm{d} x$

$$
+\frac{4}{\pi} \int_{\frac{3 \pi}{8}}^{\frac{\pi}{2}} \ln \mathcal{T}_{*}(x, \alpha) \Omega(x) \mathrm{d} x
$$

$\frac{1}{\vartheta-u} \int_{u}^{\vartheta}\left[\ln \mathcal{T}^{*}(x, \alpha)\right] \Omega(x) \mathrm{d} x=\frac{4}{\pi} \int_{\frac{\pi}{4}}^{\frac{\pi}{2}}\left[\ln \mathcal{T}^{*}(x, \alpha)\right] \Omega(x) \mathrm{d} x=\frac{4}{\pi} \int_{\frac{\pi}{4}}^{\frac{3 \pi}{8}}\left[\ln \mathcal{T}^{*}(x, \alpha)\right] \Omega(x) \mathrm{d} x$

$$
+\frac{4}{\pi} \int_{\frac{3 \pi}{8}}^{\frac{\pi}{2}} \ln \mathcal{T}^{*}(x, \alpha) \Omega(x) \mathrm{d} x
$$

$=\frac{4}{\pi} \int_{\frac{\pi}{4}}^{\frac{3 \pi}{8}}\left[\ln \left(\alpha \mathrm{e}^{\sin x}\right)\right]\left(x-\frac{\pi}{4}\right) \mathrm{d} x+\frac{4}{\pi} \int_{\frac{3 \pi}{8}}^{\frac{\pi}{2}}\left[\ln \left(\alpha \mathrm{e}^{\sin x}\right)\right]\left(\frac{\pi}{2}-x\right) \mathrm{d} x \approx \frac{1}{25 \pi}\left[\frac{31}{2} \ln (\alpha)+14\right]$,
$=\frac{4}{\pi} \int_{\frac{\pi}{4}}^{\frac{3 \pi}{8}}\left[\ln (2-\alpha) \mathrm{e}^{\sin x}\right]\left(x-\frac{\pi}{2}\right) \mathrm{d} x+\frac{4}{\pi} \int_{\frac{3 \pi}{8}}^{\frac{3 \pi}{2}}\left[\ln \left((2-\alpha) \mathrm{e}^{\sin x}\right)\right]\left(\frac{\pi}{2}-x\right) \mathrm{d} x \approx \frac{1}{25 \pi}\left[\frac{31}{2} \ln (2-\alpha)+14\right]$,

and

$\ln \left[\mathcal{T}_{*}(u, \alpha) \times \mathcal{T}_{*}(\vartheta, \alpha)\right] \int_{0}^{1} \tau^{s} \Omega((1-\tau) u+\tau \vartheta) \mathrm{d} \tau$

$\ln \left[\mathcal{T}^{*}(u, \alpha) \times \mathcal{T}^{*}(\vartheta, \alpha)\right] \int_{0}^{1} \tau^{s} \Omega((1-\tau) u+\tau \vartheta) \mathrm{d} \tau$

$=\left[2 \ln (\alpha)+\frac{2+\sqrt{2}}{2}\right]\left[\int_{0}^{\frac{1}{2}} \tau^{2} \mathrm{~d} x+\int_{\frac{1}{2}}^{1} \tau(1+\tau) \mathrm{d} \tau\right] \approx \frac{17}{24 \pi}\left[\frac{63}{10} \ln (\alpha)+\frac{2+\sqrt{2}}{2}\right]$,

$=\left[2 \ln (2-\alpha)+\frac{2+\sqrt{2}}{2}\right]\left[\int_{0}^{\frac{1}{2}} \tau^{2} \mathrm{~d} x+\int_{\frac{1}{2}}^{1} \tau(1+\tau) \mathrm{d} \tau\right] \approx \frac{17}{24 \pi}\left[\frac{63}{10} \ln (2-\alpha)+\frac{2+\sqrt{2}}{2}\right]$.

From (41) and (42), we have

$\left[\frac{1}{25 \pi}\left[\frac{31}{2} \ln (\alpha)+14\right], \frac{1}{25 \pi}\left[\frac{31}{2} \ln (2-\alpha)+14\right]\right] \leq_{l}\left[\frac{17}{24 \pi}\left[\frac{63}{10} \ln (\alpha)+\frac{2+\sqrt{2}}{2}\right], \frac{17}{24 \pi}\left[\frac{63}{10} \ln (2-\alpha)+\frac{2+\sqrt{2}}{2}\right]\right]$,

for all $\alpha \in(0,1]$. Hence, Theorem 3.6 is verified.

For Theorem 3.7, we have

$\ln \mathcal{T}_{*}\left(\frac{u+\vartheta}{2}, \alpha\right)=\ln \mathcal{T}_{*}\left(\frac{3 \pi}{8}, \alpha\right) \approx \ln \left(\frac{5}{2} \alpha\right)$
$\ln \mathcal{T}^{*}\left(\frac{u+\vartheta}{2}, \alpha\right)=\ln \mathcal{T}^{*}\left(\frac{3 \pi}{8}, \alpha\right) \approx \ln \left(\frac{5}{2}(2-\alpha)\right)$

$\int_{u}^{\vartheta} \Omega(x) \mathrm{d} x=\int_{\frac{\pi}{4}}^{\frac{3 \pi}{8}}\left(x-\frac{\pi}{4}\right) \mathrm{d} x+\int_{\frac{3 \pi}{8}}^{\frac{\pi}{2}}\left(\frac{\pi}{2}-x\right) \mathrm{d} x \approx \frac{3}{20}$ $\frac{2^{1-s}}{\int_{u}^{\vartheta} \Omega(x) \mathrm{d} x} \int_{u}^{\vartheta}\left[\ln \mathcal{T}_{*}(x, \alpha)\right] \Omega(x) \mathrm{d} x \approx \frac{2}{15}\left[\frac{31}{4} \ln (\alpha)+7\right]$

$\frac{2^{1-s}}{\int_{u}^{\vartheta} \Omega(x) \mathrm{d} x} \int_{u}^{\vartheta}\left[\ln \mathcal{T}^{*}(x, \alpha)\right] \Omega(x) \mathrm{d} x \approx \frac{2}{15}\left[\frac{31}{4} \ln (2-\alpha)+7\right]$

From (43) and (44), we have

$$
\begin{aligned}
& {\left[\ln \left(\frac{5}{2} \alpha\right), \ln \left(\frac{5}{2}(2-\alpha)\right)\right]} \\
& \quad \leq{ }_{l}\left[\frac{2}{15}\left[\frac{31}{4} \ln (\alpha)+7\right], \frac{2}{15}\left[\frac{31}{4} \ln (2-\alpha)+7\right]\right] .
\end{aligned}
$$

Hence, Theorem 3.7 is verified. 
Now, we prove the Jensen's inequality for log-s-convex fuzzy-IVF in the second sense.

Theorem $\quad 3.10$ Let $\omega_{j} \in \mathbb{R}^{+}, \quad x_{j} \in[u, \vartheta]$, $(j=1,2,3, \ldots k, k \geq 2)$ and $\mathcal{T}:[u, \vartheta] \rightarrow \mathbb{F}_{C}(\mathbb{R})$ be a log$s$-convex fuzzy-IVF in the second sense, whose $\alpha$-levels define the family of IVFs $\mathcal{T}_{\alpha}:[u, \vartheta] \subset \mathbb{R} \rightarrow \mathcal{K}_{C}^{+}$are given by $\mathcal{T}_{\alpha}(x)=\left[\mathcal{T}_{*}(x, \alpha), \mathcal{T}^{*}(x, \alpha)\right]$ for all $x \in[u, \vartheta]$ and for all $\alpha \in(0,1]$. Then,

$\mathcal{T}\left(\frac{1}{W_{k}} \sum_{j=1}^{k} \omega_{j} x_{j}\right) \preccurlyeq \prod_{j=1}^{k}\left[\mathcal{T}\left(x_{j}\right)\right]^{\left(\frac{\omega_{j}}{w_{k}}\right)^{s}}$,

where $W_{k}=\sum_{j=1}^{k} \omega_{j}$. If $\mathcal{T}$ is log-s-concave in the second sense then, inequality (45) is reversed.

Proof When $k=2$ inequality (45) is true. Consider inequality (16) is true for $k=n-1$, then

$\mathcal{T}\left(\frac{1}{W_{n-1}} \sum_{j=1}^{n-1} \omega_{j} x_{j}\right) \preccurlyeq \prod_{j=1}^{n-1}\left[\mathcal{T}\left(x_{j}\right)\right]^{\left(\frac{\omega_{j}}{w_{n-1}}\right)^{s}}$,

Now, let us prove that inequality (45) holds for $k=n$ :

$$
\begin{aligned}
\mathcal{T} & \left.\frac{1}{W_{n}} \sum_{j=1}^{n} \omega_{j} x_{j}\right) \\
& =\mathcal{T}\left(\frac{1}{W_{n-2}} \sum_{j=1}^{n-2} \omega_{j} x_{j}+\frac{\omega_{n-1}+\omega_{n}}{W_{n}}\left(\frac{\omega_{n-1}}{\omega_{n-1}+\omega_{n}} x_{n-1}+\frac{\omega_{n}}{\omega_{n-1}+\omega_{n}} x_{n}\right)\right) .
\end{aligned}
$$

Therefore, for every $\alpha \in(0,1]$, we have

$$
\begin{aligned}
& \mathcal{T}_{*}\left(\frac{1}{W_{n}} \sum_{j=1}^{n} \omega_{j} x_{j}, \alpha\right) \\
& \mathcal{T}^{*}\left(\frac{1}{W_{n}} \sum_{j=1}^{n} \omega_{j} x_{j}, \alpha\right) \\
& \leq \mathcal{T}_{*}\left(\frac{1}{W_{n}} \sum_{j=1}^{n-2} \omega_{j} x_{j}+\frac{\omega_{n-1}+\omega_{n}}{W_{n}}\left(\frac{\omega_{n-1}}{\omega_{n-1}+\omega_{n}} x_{n-1}+\frac{\omega_{n}}{\omega_{n-1}+\omega_{n}} x_{n}, \alpha\right)\right), \\
& \leq \mathcal{T}^{*}\left(\frac{1}{W_{n}} \sum_{j=1}^{n-2} \omega_{j} x_{j}+\frac{\omega_{n-1}+\omega_{n}}{W_{n}}\left(\frac{\omega_{n-1}}{\omega_{n-1}+\omega_{n}} x_{n-1}+\frac{\omega_{n}}{\omega_{n-1}+\omega_{n}} x_{n}, \alpha\right)\right),
\end{aligned}
$$$$
\leq\left[\prod_{j=1}^{n-2}\left[\mathcal{T}_{*}\left(x_{j}, \alpha\right)\right]^{\left(\frac{\omega_{j}}{W_{n}}\right)^{s}} \mathcal{T}_{*}\left(\frac{\omega_{n-1}}{\omega_{n-1}+\omega_{n}} x_{n-1}+\frac{\omega_{n}}{\omega_{n-1}+\omega_{n}} x_{n}, \alpha\right)\right]^{\left(\frac{\omega_{n-1}+\omega_{n}}{w_{n}}\right)^{s}}
$$$$
\leq \prod_{j=1}^{n-2}\left[\mathcal{T}^{*}\left(x_{j}, \alpha\right)\right]^{\left(\frac{\omega_{j}}{w_{n}}\right)^{s}}\left[\mathcal{T}^{*}\left(\frac{\omega_{n-1}}{\omega_{n-1}+\omega_{n}} x_{n-1}+\frac{\omega_{n}}{\omega_{n-1}+\omega_{n}} x_{n}, \alpha\right)\right]^{\left(\frac{\omega_{n-1}+\omega_{n}}{w_{n}}\right)^{s}},
$$

$$
\begin{aligned}
& \leq \prod_{j=1}^{n-2}\left[\mathcal{T}_{*}\left(x_{j}, \alpha\right)\right]^{\left(\frac{\omega_{j}}{w_{n}}\right)^{s}}\left[\left[\mathcal{T}_{*}\left(x_{n-1}, \alpha\right)\right]^{\left(\frac{\omega_{n-1}}{\omega_{n-1}+\omega_{n}}\right)^{s}}\left[\mathcal{T}_{*}\left(x_{n}, \alpha\right)\right]^{\left(\frac{\omega_{n}}{\omega_{n-1}+\omega_{n}}\right)^{s}}\right]^{\left(\frac{\omega_{n-1}+\omega_{n}}{w_{n}}\right)^{s},} \\
& \leq \prod_{j=1}^{n-2}\left[\mathcal{T}^{*}\left(x_{j}, \alpha\right)\right]^{\left(\frac{\omega_{j}}{w_{n}}\right)^{s}}\left[\left[\mathcal{T}^{*}\left(x_{n-1}, \alpha\right)\right]^{\left(\frac{\omega_{n-1}}{\omega_{n-1}+\omega_{n}}\right)^{s}}\left[\mathcal{T}^{*}\left(x_{n}, \alpha\right)\right]^{\left(\frac{\omega_{n}}{\omega_{n-1}+\omega_{n}}\right)^{s}}\right]^{\left(\frac{\omega_{n-1}+\omega_{n}}{w_{n}}\right)^{s},}
\end{aligned}
$$

To obtain a refinement of Jensen's inequality for log$s$-convex-IVFs firstly, we prove the following the result:

Theorem 3.12 Let $s \in(0,1)$ and $\mathcal{T}:[u, \vartheta] \rightarrow \mathbb{F}_{C}(\mathbb{R})$ be a fuzzy-IVF, whose $\alpha$-levels define the family of IVFs $\mathcal{T}_{\alpha}:[u, \vartheta] \subset \mathbb{R} \rightarrow \mathcal{K}_{C}^{+}$are given by $\mathcal{T}_{\alpha}(x)=\left[\mathcal{T}_{*}(x, \alpha), \mathcal{T}^{*}(x, \alpha)\right]$ 
for all $x \in[u, \vartheta]$ and for all $\alpha \in(0,1]$. If $(L, U) \subseteq[u, \vartheta]$ and $\mathcal{T}$ be a log-s-convex fuzzy-IVF in the second sense then, for $x_{1}, x_{2}, x_{3} \in[u, \vartheta], x_{1}<x_{2}<x_{3}$ such that $x_{3}-x_{1}, x_{3}-x_{2}$, $x_{2}-x_{1} \in[0,1]$, we have

$\left(x_{3}-x_{1}\right)^{s} \mathcal{T}\left(x_{2}\right) \leqslant \mathcal{T}\left(x_{1}\right)^{\left(x_{3}-x_{2}\right)^{s}} \mathcal{T}\left(x_{3}\right)^{\left(x_{2}-x_{1}\right)^{s}}$.

Proof Let $x_{1}, x_{2}, x_{3} \in[u, \vartheta]$ and $\left(x_{3}-x_{2}\right)^{s}>0$. Then by hypothesis, we have

$\left(\frac{x_{3}-x_{2}}{x_{3}-x_{1}}\right)^{s}=\frac{\left(x_{3}-x_{2}\right)^{s}}{\left(x_{3}-x_{1}\right)^{s}}$ and $\left(\frac{x_{2}-x_{1}}{x_{3}-x_{1}}\right)^{s}=\frac{\left(x_{2}-x_{1}\right)^{s}}{\left(x_{3}-x_{1}\right)^{s}}$.

Consider $\lambda=\frac{x_{3}-x_{2}}{x_{3}-x_{1}}$, then $x_{2}=\lambda x_{1}+(1-\lambda) x_{3}$. Since $\mathcal{T}$ is a log-s-convex fuzzy-IVF then, by hypothesis, we have

$$
\begin{aligned}
& \mathcal{T}_{*}\left(x_{2}, \alpha\right) \leq\left[\mathcal{T}_{*}\left(x_{1}, \alpha\right)\right]^{\left(\frac{x_{3}-x_{2}}{x_{3}-x_{1}}\right)^{s}}\left[\mathcal{T}_{*}\left(x_{3}, \alpha\right)\right]^{\left(\frac{x_{2}-x_{1}}{x_{3}-x_{1}}\right)^{s},} \\
& \mathcal{T}^{*}\left(x_{2}, \alpha\right) \leq\left[\mathcal{T}^{*}\left(x_{1}, \alpha\right)\right]^{\left(\frac{x_{3}-x_{2}}{x_{3}-x_{1}}\right)^{s}}\left[\mathcal{T}^{*}\left(x_{3}, \alpha\right)\right]^{\left(\frac{x_{2}-x_{1}}{x_{3}-x_{1}}\right)^{s}}, \\
& =\left[\mathcal{T}_{*}\left(x_{1}, \alpha\right)\right]^{\frac{\left(x_{3}-x_{2}\right)^{s}}{\left(x_{3}-x_{1}\right)^{s}}}\left[\mathcal{T}_{*}\left(x_{3}, \alpha\right)\right]^{\frac{\left(x_{2}-x_{1}\right)^{s}}{\left(x_{3}-x_{1}\right)^{s}}}, \\
& =\left[\mathcal{T}^{*}\left(x_{1}, \alpha\right)\right]^{\frac{\left(x_{3}-x_{2}\right)^{s}}{\left(x_{3}-x_{1}\right)^{s}}}\left[\mathcal{T}^{*}\left(x_{3}, \alpha\right)\right]^{\frac{\left(x_{2}-x_{1}\right)^{s}}{\left(x_{3}-x_{1}\right)^{s}}} .
\end{aligned}
$$

Taking log on both sides of (49), we have

$$
\begin{aligned}
& \left.\mathcal{T}_{*}\left(x_{2}, \alpha\right)\right)^{\left(x_{3}-x_{1}\right)^{s}} \leq\left[\mathcal{T}_{*}\left(x_{1}, \alpha\right)\right]^{\left(x_{3}-x_{2}\right)^{s}}\left[\mathcal{T}_{*}\left(x_{3}, \alpha\right)\right]^{\left(x_{2}-x_{1}\right)^{s}}, \\
& \mathcal{T}^{*}\left(x_{2}, \alpha\right)^{\left(x_{3}-x_{1}\right)^{s}} \leq\left[\mathcal{T}^{*}\left(x_{1}, \alpha\right)\right]^{\left(x_{3}-x_{2}\right)^{s}}\left[\mathcal{T}^{*}\left(x_{3}, \alpha\right)\right]^{\left(x_{2}-x_{1}\right)^{s}},
\end{aligned}
$$

that is

$$
\begin{aligned}
& {\left[\left[\mathcal{T}_{*}\left(x_{2}, \alpha\right)\right]^{\left(x_{3}-x_{1}\right)^{s}},\left[\mathcal{T}^{*}\left(x_{2}, \alpha\right)\right]^{\left(x_{3}-x_{1}\right)^{s}}\right]} \\
& \leq_{p}\left[\left[\mathcal{T}_{*}\left(x_{1}, \alpha\right)\right]^{\left(x_{3}-x_{2}\right)^{s}}\left[\mathcal{T}_{*}\left(x_{3}, \alpha\right)\right]^{\left(x_{2}-x_{1}\right)^{s}},\left[\mathcal{T}^{*}\left(x_{1}, \alpha\right)\right]^{\left(x_{3}-x_{2}\right)^{s}}\left[\mathcal{T}^{*}\left(x_{3}, \alpha\right)\right]^{\left(x_{2}-x_{1}\right)^{s}}\right] \text {. }
\end{aligned}
$$

Theorem 3.13 Let $\omega_{j} \in \mathbb{R}^{+}, \quad x_{j} \in[u, \vartheta]$, $(j=1,2,3, \ldots k, k \geq 2)$ and $\mathcal{T}:[u, \vartheta] \rightarrow \mathbb{F}_{C}(\mathbb{R})$ be a logs-convex fuzzy-IVF in the second sense, whose $\alpha$-levels define the family of IVFs $\mathcal{T}_{\alpha}:[u, \vartheta] \subset \mathbb{R} \rightarrow \mathcal{K}_{C}^{+}$are given by $\mathcal{T}_{\alpha}(x)=\left[\mathcal{T}_{*}(x, \alpha), \mathcal{T}^{*}(x, \alpha)\right]$ for all $x \in[u, \vartheta]$ and for all $\alpha \in(0,1]$. If $(L, U) \subseteq[u, \vartheta]$ then,

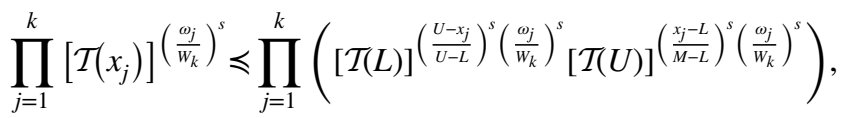

where $W_{k}=\sum_{j=1}^{k} \omega_{j}$. If $\mathcal{T}$ is log-s-concave in the second sense then, inequality (50) is reversed.

Proof Consider $=x_{1}, x_{j}=x_{2},(j=1,2,3, \ldots k), U=x_{3}$ in inequality (50). Then, for each $\alpha \in(0,1]$, then

$$
\begin{aligned}
& \mathcal{T}_{*}\left(x_{j}, \alpha\right) \leq\left[\mathcal{T}_{*}(L, \alpha)\right]^{\left(\frac{U-x_{j}}{U-L}\right)^{s}}\left[\mathcal{T}_{*}(U, \alpha)\right]^{\left(\frac{x_{j}-L}{M-L}\right)^{s},} \\
& \mathcal{T}^{*}\left(x_{j}, \alpha\right) \leq\left[\mathcal{T}^{*}(L, \alpha)\right]^{\left(\frac{U-x_{j}}{U-L}\right)^{s}}\left[\mathcal{T}^{*}(U, \alpha)\right]^{\left(\frac{x_{j}-L}{M-L}\right)^{s}} .
\end{aligned}
$$

Above inequality can be written as,

$\mathcal{T}_{*}\left(x_{j}, \alpha\right)^{\left(\frac{\omega_{j}}{w_{k}}\right)^{s}} \leq\left[\mathcal{T}_{*}(L, \alpha)\right]^{\left(\frac{U-x_{j}}{U-L}\right)^{s}\left(\frac{\omega_{j}}{W_{k}}\right)^{s}}\left[\mathcal{T}_{*}(U, \alpha)\right]^{\left(\frac{x_{j}-L}{M-L}\right)^{s}\left(\frac{\omega_{j}}{W_{k}}\right)^{s},}$
$\mathcal{T}^{*}\left(x_{j}, \alpha\right)^{\left(\frac{\omega_{j}}{W_{k}}\right)^{s}} \leq\left[\mathcal{T}^{*}(L, \alpha)\right]^{\left(\frac{U-x_{j}}{U-L}\right)^{s}\left(\frac{\omega_{j}}{W_{k}}\right)^{s}}\left[\mathcal{T}^{*}(U, \alpha)\right]^{\left(\frac{x_{j}-L}{M-L}\right)^{s}\left(\frac{\omega_{j}}{W_{k}}\right)^{s}}$.

Taking multiplication of all inequalities (51) for $j=1,2,3, \ldots k$, we have
Hence

$$
\left[\mathcal{T}\left(x_{2}\right)\right]^{\left(x_{3}-x_{1}\right)^{s}} \preccurlyeq\left[\mathcal{T}\left(x_{1}\right)\right]^{\left(x_{3}-x_{2}\right)^{s}}\left[\mathcal{T}\left(x_{3}\right)\right]^{\left(x_{2}-x_{1}\right)^{s}} .
$$

$$
\begin{gathered}
\prod_{j=1}^{k} \mathcal{T}_{*}\left(x_{j}, \alpha\right)^{\left(\frac{\omega_{j}}{w_{k}}\right)^{s}} \\
\leq \prod_{j=1}^{k}\left(\left[\mathcal{T}_{*}(L, \alpha)\right]^{\left(\frac{U-x_{j}}{U-L}\right)^{s}\left(\frac{\omega_{j}}{w_{k}}\right)^{s}}\left[\mathcal{T}_{*}(U, \alpha)\right]^{\left.\left(\frac{x_{j}-L}{M-L}\right)^{s}\left(\frac{\omega_{j}}{w_{k}}\right)^{s}\right),}\right. \\
\prod_{j=1}^{k} \mathcal{T}^{*}\left(x_{j}, \alpha\right)^{\left(\frac{\omega_{j}}{w_{k}}\right)^{s}} \\
\leq \prod_{j=1}^{k}\left(\left[\mathcal{T}^{*}(L, \alpha)\right]^{\left(\frac{U-x_{j}}{U-L}\right)^{s}\left(\frac{\omega_{j}}{w_{k}}\right)^{s}}\left[\mathcal{T}^{*}(U, \alpha)\right]^{\left.\left(\frac{x_{j}-L}{M-L}\right)^{s}\left(\frac{\omega_{j}}{w_{k}}\right)^{s}\right),}\right.
\end{gathered}
$$

that is 


$$
\begin{aligned}
& \prod_{j=1}^{k} \mathcal{T}_{\alpha}\left(x_{j}\right)^{\left(\frac{\omega_{j}}{W_{k}}\right)^{s}}=\left[\prod_{j=1}^{k} \mathcal{T}_{*}\left(x_{j}, \alpha\right)^{\left(\frac{\omega_{j}}{W_{k}}\right)^{s}}, \prod_{j=1}^{k} \mathcal{T}^{*}\left(x_{j}, \alpha\right)^{\left(\frac{\omega_{j}}{W_{k}}\right)^{s}}\right]
\end{aligned}
$$

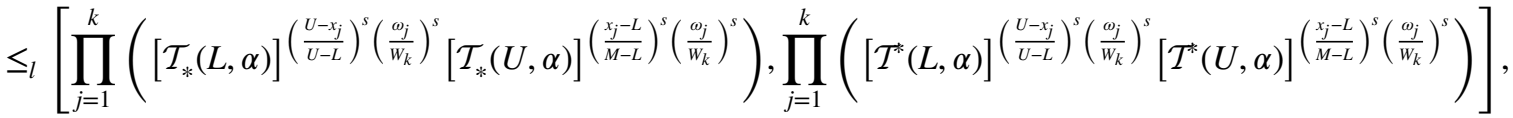

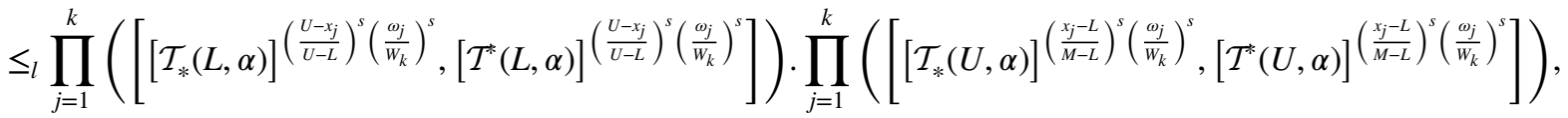

$$
\begin{aligned}
& =\prod_{j=1}^{k}\left[\mathcal{T}_{\alpha}(L)\right]^{\left(\frac{U-x_{j}}{U-L}\right)^{s}\left(\frac{\omega_{j}}{W_{k}}\right)^{s}} \cdot \prod_{j=1}^{k}\left[\mathcal{T}_{\alpha}(U)\right]^{\left(\frac{x_{j}-L}{M-L}\right)^{s}\left(\frac{\omega_{j}}{W_{k}}\right)^{s}} .
\end{aligned}
$$

Thus,

$$
\prod_{j=1}^{k}\left[\mathcal{T}\left(x_{j}\right)\right]^{\left(\frac{\omega_{j}}{w_{k}}\right)^{s}} \leqslant \prod_{j=1}^{k}\left([\mathcal{T}(L)]^{\left(\frac{U-x_{j}}{U-L}\right)^{s}\left(\frac{\omega_{j}}{w_{k}}\right)^{s}}[\mathcal{T}(U)]^{\left(\frac{x_{j}-L}{M-L}\right)^{s}\left(\frac{\omega_{j}}{w_{k}}\right)^{s}}\right),
$$

this completes the proof.

\section{Remark 3.14}

(i) If $s=1$, then Theorems 3.12 and 3.13 reduces to the result for log convex fuzzy-IVF.

(ii) If $\mathcal{T}_{*}(u, \alpha)=\mathcal{T}^{*}(\vartheta, \alpha)$ with $\alpha=1$, then Theorems 3.12 and 3.13 reduce to the result for log-s-convex function in the second sense, see [6].

(iii) If $\mathcal{T}_{*}(u, \alpha)=\mathcal{T}^{*}(\vartheta, \alpha)$ with $\alpha=1$ and $s=1$, then Theorems 3.12 and 3.13 reduce to the result for logconvex function, see [13].

\section{Results and discussion}

We derive some Jensen, Schur and HH-Inequalities for log-s-convex fuzzy-IVFs in the second sense by means of fuzzy order relation. Our results generalize most of the results which are proved for classical log-convex functions. We would like to mention that due to the lack of "fuzzy interval derivatives" with some good properties, we have not discussed those inequalities involving fuzzy interval derivatives.

\section{Conclusion}

$\mathrm{HH}$-Inequalities are true for this concept of log-s-convex fuzzy-IVFs in the second sense. As a future research, we try to explore this concept for generalized of log-s-convex fuzzy-IVFs in the second sense and some applications in fuzzy-interval nonlinear programing. We hope that this concept will be helpful for other authors to pay their roles in different fields of sciences.

Acknowledgements The authors would like to thank the Rector, COMSATS University Islamabad, Islamabad, Pakistan, for providing excellent research and academic environments.

Author contributions All authors contributed equally to the writing of this paper. All authors read and approved the final manuscript.

Funding This study was funded by National Natural Science Foundation of China (no. 71771140).

Data availability No data were used to support this study.

\section{Declarations}

Conflict of interest The authors declare that they have no competing interests.

\section{References}

1. Alomari M, Darus M, Dragomir SS, Cerone P (2010) Ostrowski type inequalities for functions whose derivatives are s-convex in the second sense. Appl Math Lett 23:1071-1076

2. Cerone P, Dragomir SS (2004) Ostrowski type inequalities for functions whose derivatives satisfy certain convexity assumptions. Demonstr Math 37(2):299-308

3. Kaleva O (1987) Fuzzy differential equations. Fuzzy Sets Syst 24:301-317

4. Hadamard J (1893) Étude sur les propriétés des fonctions entières et en particulier d'une fonction considérée par Riemann. Journal de mathématiques pures et appliquées 7:171-215

5. Hermite C (1883) Sur deux limites d'une intégrale définie. Mathesis 3:82-97

6. Varošanec S (2007) On h-convexity. J Math Anal Appl 326(1):303-311

7. Xi BY, Qi F (2015) Some integral inequalities of Hermite-Hadamard type for $s$-logarithmically convex functions. Acta Math Sci Ser A (Chin Ed) 35(3):515-524

8. Noor MA (2007) Hermite-Hadamard integral inequalities for logpreinvex functions. J Math Anal Approx Theory 2(2):126-131

9. Pečarić JE, Proschan F, Tong YL (1991) Convex functions, partial orderingsand statistical applications. Academic Press 
10. Noor MA, Qi F, Awan MU (2013) Some Hermite-Hadamard type inequalities for log- $h$-convex functions. Analysis 33(4):367-375

11. Adil Khan M, Pečarić J, Chu Y-M (2020) Refinements of Jensen's and McShane's inequalities with applications. AIMS Math 5(5):4931-4945

12. Dragomir SS (2001) Refinements of the Hermite-Hadamard integral inequality for log-convex functions. Aust Math Soc Gaz 28(3):129-134

13. Dragomir SS (2011) A survey of Jensen type inequalities for logconvex functions of selfadjoint operators in Hilbert spaces. Commun Math Anal 10(1):82-104

14. Dragomir SS, Mond B (1998) Integral inequalities of Hadamard type for log convexfunctions. Demonstr Math 31(2):354-364

15. Dragomir SS (2017) New inequalities of Hermite-Hadamard type for log convex functions. Khayyam J Math 3(2):98-115

16. Niculescu CP (2012) The Hermite-Hadamard inequality for $\log$ convex functions. Nonlinear Anal 75(2):662-669

17. Moore RE (1966) Interval analysis. Prentice Hall, Englewood Cliffs

18. Kulish UW, Miranker W (1981) Computer arithmetic in theory and practice. Academic Press, New York

19. Guo Y, Ye G, Zhao D, Liu W (2019) Some integral inequalities for log- $h$-convex interval-valued functions. IEEE Access $7: 86739-86745$

20. Chalco-Cano Y, Flores-Franulič A, Román-Flores H (2012) Ostrowski type inequalities for interval-valued functions using generalized Hukuhara derivative. Comput Appl Math 31(3):457-472

21. Chalco-Cano Y, Lodwick WA, Condori-Equice W (2015) Ostrowski type inequalities and applications in numerical integration for interval-valued functions. Soft Comput 19:3293-3300

22. Costa TM, Román-Flores H, Chalco-Cano Y (2019) Opialtype inequalities for interval-valued functions. Fuzzy Sets Syst 358:48-63

23. Dragomir SS, Pearce CEM (2000) Selected topics on HermiteHadamard inequalities and applications. RGMIA Monographs Victoria University

24. Román-Flores H, Chalco-Cano Y, Lodwick WA (2018) Some integral inequalities for interval-valued functions. Comput Appl Math 37(2):1306-1318

25. Zhao DF, An TQ, Ye GJ, Liu W (2018) New Jensen and Hermite-Hadamard type inequalities for $h$-convex interval-valued functions. J Inequal Appl 2018:1-14
26. Zadeh LA (1965) Fuzzy sets. Inf Control 8(3):338-353

27. Nanda S, Kar K (1992) Convex fuzzy mappings. Fuzzy Sets Syst 48:129-132

28. Chang SS (1991) Variational inequality and complementarity problems theory and applications. Shanghai Scientific and Technological Literature Publishing House, Shanghai

29. Noor MA (1994) Fuzzy preinvex functions. Fuzzy Sets Syst 64(1):95-104

30. Bede B, Gal SG (2005) Generalizations of the differentiability of fuzzy-number-valued functions with applications to fuzzy differential equations. Fuzzy Sets Syst 151(3):581-599

31. Bede B (2013) Mathematics of fuzzy sets and fuzzy logic. In: Studies in fuzziness and soft computing, vol. 295. Springer

32. Cervelati J, Jiménez-Gamero MD, Vilca-Labra F, Rojas-Medar MA (2004) Continuity for $s$-convex fuzzy processes. In: Soft methodology and random information systems, vol 7, pp 653-660

33. Chalco-Cano Y, Rojas-Medar MA, Román-Flores H (2000) M-convex fuzzy mappings and fuzzy integral mean. Comput Math Appl 40(10-11):1117-1126

34. Khan MB, Noor MA, Noor KI, Chu Y-M (2021) New HermiteHadamard type inequalities for $(h 1, h 2)$-convex fuzzy-intervalvalued functions. Adv Differ Equ 2021:6-20

35. Puri ML, Ralescu DA (1986) Fuzzy random variables. In: Readings in fuzzy sets for intelligent systems, vol 114, pp 409-422

36. Osuna-Gómez R, Jiménez-Gamero MD, Chalco-Cano Y, RojasMedar MA (2004) Hadamard and Jensen inequalities for $s$-convex fuzzy processes. In: Soft methodology and random information systems. Advances in soft computing, vol 126. Springer, Berlin, pp $1-15$

37. Costa TM (2017) Jensen's inequality type integral for fuzzy-interval-valued functions. Fuzzy Sets Syst 327:1-47

38. Goetschel R Jr, Voxman W (1986) Elementary fuzzy calculus. Fuzzy Sets Syst 18:31-43

39. Diamond P, Kloeden PE (1994) Metric spaces of fuzzy sets: theory and applications. World Scientific

40. Costa TM, Roman-Flores H (2017) Some integral inequalities for fuzzy-interval-valued functions. Inf Sci 420:110-125

Publisher's Note Springer Nature remains neutral with regard to jurisdictional claims in published maps and institutional affiliations. 